\title{
On Constrained Set-Valued Semi-Infinite Programming Problems with $\rho$-Cone Arcwise Connectedness
}

\author{
Koushik Das ${ }^{1}$ (D) and Savin Treanţă ${ }^{2, *(D)}$ \\ 1 Department of Mathematics, Taki Government College, Taki 743429, West Bengal, India; \\ koushikdas.maths@gmail.com \\ 2 Department of Applied Mathematics, University Politehnica of Bucharest, 060042 Bucharest, Romania \\ * Correspondence: savin.treanta@upb.ro
}

check for updates

Citation: Das, K.; Treanţă, S. On Constrained Set-Valued Semi-Infinite Programming Problems with $\rho$-Cone Arcwise Connectedness. Axioms 2021, 10, 302. https://doi.org/10.3390/ axioms10040302

Academic Editor: Martin Bohner

Received: 21 October 2021

Accepted: 10 November 2021

Published: 12 November 2021

Publisher's Note: MDPI stays neutral with regard to jurisdictional claims in published maps and institutional affiliations.

Copyright: (C) 2021 by the authors Licensee MDPI, Basel, Switzerland. This article is an open access article distributed under the terms and conditions of the Creative Commons Attribution (CC BY) license (https:// creativecommons.org/licenses/by/ $4.0 /)$.

\begin{abstract}
In this paper, we establish sufficient Karush-Kuhn-Tucker (for short, KKT) conditions of a set-valued semi-infinite programming problem (SP) via the notion of contingent epiderivative of set-valued maps. We also derive duality results of Mond-Weir (MWD), Wolfe (WD), and mixed (MD) types of the problem (SP) under $\rho$-cone arcwise connectedness assumptions.
\end{abstract}

Keywords: set-valued map; convex cone; duality; contingent epiderivative

MSC: 26B25; 49N15

\section{Introduction}

Over the last few decades, many authors, such as Hanson [1], Craven [2], Corley [3], Zalmai [4] etc., studied different types of optimization problems. One of such optimization problems is semi-infinite programming problem. In recent times, many authors, such as Goberna and Lopez [5], Shapiro [6], etc., have studied optimality conditions of semi-infinite programming problems. In 2005, Shapiro [7] studied the Lagrangian-type duality of semiinfinite programming problems under convexity assumption. In 2010, Kostyukova and Tchemisova [8] established sufficient optimality conditions of semi-infinite programming problems using convexity assumption. In 2012, Mishra and Jaiswal [9] established the sufficient optimality conditions of semi-infinite programming problems using generalized convexity assumption. They also formulated duality model and proved the corresponding theorems of Mond-Weir type dual.

In 1976, Avriel [10] introduced the notion of arcwise connectedness as a generalization of convexity. It was basically introduced by replacing the line segment and joining two points by a continuous arc. Later, Fu and Wang [11] and Lalitha et al. [12] introduced the notion of cone arcwise connected set-valued maps as an extension of the class of convex set-valued maps. Lalitha et al. [12] established the sufficient optimality conditions of setvalued optimization problems via contingent epiderivative and cone arcwise connectedness assumptions. In 2013, Yu [13] established the necessary and sufficient optimality conditions for the existence of global proper efficient points of vector optimization problems using cone arcwise connectedness. Yihong and Min [14] introduced the concept of $\alpha$-order nearly cone arcwise connected set-valued maps and derived the necessary and sufficient optimality conditions of some set-valued optimization problems. Yu [15] established the necessary and sufficient optimality conditions for the existence of global proper efficient elements in vector optimization problems. In 2018, Peng and Xu [16] introduced the notion of cone subarcwise connected set-valued maps. They also established the second-order necessary optimality conditions for global proper efficiency of set-valued optimization problems.

In this paper, we consider a set-valued semi-infinite programming problem (SP), where the objective function and constraints are set-valued maps. We are mainly interested in establishing the sufficient KKT optimality conditions of the problem (SP) in terms of 
generalized cone arcwise connectedness. We also study the duality theorems of Mond-Weir (MWD), Wolfe (WD), and mixed (MD) types associated with the problem (SP).

This paper is organized as follows. In Section 2, we state some definitions and preliminary concepts of set-valued maps. The concept of $\rho$-cone arcwise connectedness is included in Section 3. In Section 4, we establish (see Section 5) the sufficient KKT optimality conditions for the problem (SP). We also study the duality results of various types using generalized cone arcwise connectedness assumptions (see Sections 6-8). Section 9 concludes the paper.

\section{Definitions and Preliminaries}

Let $V$ be a real normed space and $\Omega$ be a nonempty subset of $V$. Then, $\Omega$ is said to be a cone if $\lambda v \in \Omega$, for all $v \in \Omega$ and $\lambda \geq 0$. Furthermore, $\Omega$ is called non-trivial if $\Omega \neq\left\{\theta_{V}\right\}$, proper if $\Omega \neq V$, pointed if $\Omega \cap(-\Omega)=\left\{\theta_{V}\right\}$, solid if int $(\Omega) \neq \varnothing$, closed if $\bar{\Omega}=\Omega$, and convex if $\lambda \Omega+(1-\lambda) \Omega \subseteq \Omega$, for all $\lambda \in[0,1]$, where $\operatorname{int}(\Omega)$ and $\bar{\Omega}$ denote the interior and closure of $\Omega$, respectively, and $\theta_{V}$ is the zero element of $V$.

Aubin $[17,18]$ introduced the notion of contingent cone in set-valued optimization theory. Moreover, Aubin [17,18] and Cambini et al. [19] introduced the notion of secondorder contingent set in a set-valued optimization theory.

Definition 1 ([17,18]). Let $V$ be a real normed space, $\varnothing \neq B \subseteq V$, and $v^{\prime} \in \bar{B}$. The contingent cone to $B$ at $v^{\prime}$ is denoted by $T\left(B, v^{\prime}\right)$ and is defined as follows: an element $v \in T\left(B, v^{\prime}\right)$ if there are sequences $\left\{\lambda_{n}\right\}$ in $\mathbb{R}$, with $\lambda_{n} \rightarrow 0^{+}$and $\left\{v_{n}\right\}$ in $V$, with $v_{n} \rightarrow v$, such that

$$
v^{\prime}+\lambda_{n} v_{n} \in B, \quad \forall n \in \mathbb{N}
$$

or, there exist sequences $\left\{t_{n}\right\}$ in $\mathbb{R}$, with $t_{n}>0$ and $\left\{v_{n}^{\prime}\right\}$ in $B$, with $v_{n}^{\prime} \rightarrow v^{\prime}$, such that

$$
t_{n}\left(v_{n}^{\prime}-v^{\prime}\right) \rightarrow v
$$

Let $U, V$ be real normed spaces, $2^{V}$ be the set of all subsets of $V$, and $\Omega$ be a solid pointed convex cone in $V$. Let $\mathcal{F}: U \rightarrow 2^{V}$ be a set-valued map from $U$ to $V$, i.e., $\mathcal{F}(u) \subseteq V$, for all $u \in U$. The domain, image, graph, and epigraph of $\mathcal{F}$ are defined by

$$
\begin{gathered}
\operatorname{dom}(\mathcal{F})=\{u \in U: \mathcal{F}(u) \neq \varnothing\}, \\
\mathcal{F}(A)=\bigcup_{u \in A} \mathcal{F}(u), \text { for any } \varnothing \neq A \subseteq U, \\
\operatorname{gr}(\mathcal{F})=\{(u, v) \in U \times V: v \in \mathcal{F}(u)\},
\end{gathered}
$$

and

$$
\operatorname{epi}(\mathcal{F})=\{(u, v) \in U \times V: v \in \mathcal{F}(u)+\Omega\} .
$$

Jahn and Rauh [20] introduced the notion of contingent epiderivative of set-valued maps. It plays a vital role in various aspects of set-valued optimization problems.

Definition 2 ([20]). A single-valued map $D_{\uparrow} \mathcal{F}\left(u^{\prime}, v^{\prime}\right): U \rightarrow V$ whose epigraph coincides with the contingent cone to the epigraph of $\mathcal{F}$ at $\left(u^{\prime}, v^{\prime}\right)$, i.e.,

$$
\operatorname{epi}\left(D_{\uparrow} \mathcal{F}\left(u^{\prime}, v^{\prime}\right)\right)=T\left(\operatorname{epi}(\mathcal{F}),\left(u^{\prime}, v^{\prime}\right)\right),
$$

is said to be the contingent epiderivative of $\mathcal{F}$ at $\left(u^{\prime}, v^{\prime}\right)$.

We now turn our attention to the notion of cone convexity of set-valued maps which was introduced by Borwein [21] in 1977. 
Definition 3 ([21]). Let $A$ be a nonempty convex subset of a real normed space $U$. A set-valued map $\mathcal{F}: U \rightarrow 2^{V}$, with $A \subseteq \operatorname{dom}(\mathcal{F})$, is called $\Omega$-convex on $A$ if $\forall u_{1}, u_{2} \in A$ and $\lambda \in[0,1]$,

$$
\lambda \mathcal{F}\left(u_{1}\right)+(1-\lambda) \mathcal{F}\left(u_{2}\right) \subseteq \mathcal{F}\left(\lambda u_{1}+(1-\lambda) u_{2}\right)+\Omega .
$$

In 1976, Avriel [10] introduced the notion of arcwise connectedness of set-valued maps.

Definition 4. A subset $A$ of a real normed space $U$ is said to be an arcwise connected set if for all $u_{1}, u_{2} \in A$ there is a continuous arc $\mathcal{H}_{u_{1}, u_{2}}(\lambda)$ defined on $[0,1]$ with a value in $A$ such that $\mathcal{H}_{u_{1}, u_{2}}(0)=u_{1}$ and $\mathcal{H}_{u_{1}, u_{2}}(1)=u_{2}$.

Fu and Wang [11] and Lalitha et al. [12] introduced the notion of cone arcwise connectedness of set-valued maps. It is basically an extension of the class of cone convex set-valued maps.

Definition 5 ([11,12]). Let $A$ be an arcwise connected subset of a real normed space $U$ and $\mathcal{F}: U \rightarrow 2^{V}$ be a set-valued map, with $A \subseteq \operatorname{dom}(\mathcal{F})$. Then, $\mathcal{F}$ is said to be $\Omega$-arcwise connected on $A$ if

$$
(1-\lambda) \mathcal{F}\left(u_{1}\right)+\lambda \mathcal{F}\left(u_{2}\right) \subseteq \mathcal{F}\left(\mathcal{H}_{u_{1}, u_{2}}(\lambda)\right)+\Omega, \quad \forall u_{1}, u_{2} \in A \text { and } \forall \lambda \in[0,1] .
$$

Peng and $\mathrm{Xu}$ [16] introduced the notion of cone subarcwise connectedness of setvalued maps.

Definition 6 ([16]). Let $A$ be an arcwise connected subset of a real normed space $U, e \in \operatorname{int}(\Omega)$, and $\mathcal{F}: U \rightarrow 2^{V}$ be a set-valued map, with $A \subseteq \operatorname{dom}(\mathcal{F})$. Then, $\mathcal{F}$ is said to be $\Omega$-subarcwise connected on $A$ if

$$
\begin{array}{r}
(1-\lambda) \mathcal{F}\left(u_{1}\right)+\lambda \mathcal{F}\left(u_{2}\right)+\epsilon e \subseteq \mathcal{F}\left(\mathcal{H}_{u_{1}, u_{2}}(\lambda)\right)+\Omega, \\
\forall u_{1}, u_{2} \in A, \forall \epsilon>0, \text { and } \forall \lambda \in[0,1] .
\end{array}
$$

\section{3. $\rho$-Cone Arcwise Connectedness}

Das and Nahak [22-25] and Treanţă and Das [26] introduced the notion of $\rho$-cone convexity of set-valued maps. They establish the sufficient KKT optimality conditions of set-valued optimization problems under contingent epiderivative and $\rho$-cone convexity assumptions. They also construct various duality models and prove the associated duality theorems. For $\rho=0$, we get the usual notion of cone convexity of set-valued maps introduced by Borwein [21] in the year of 1977.

We introduce the notion of $\rho$-cone arcwise connectedness of set-valued maps as a generalization of cone arcwise connected set-valued maps.

Definition 7. Let $A$ be an arcwise connected subset of a real normed space $U, e \in \operatorname{int}(\Omega)$, and $\mathcal{F}: U \rightarrow 2^{V}$ be a set-valued map, with $A \subseteq \operatorname{dom}(\mathcal{F})$. Then, $\mathcal{F}$ is said to be $\rho$ - $\Omega$-arcwise connected on $A$ with respect to e if there exists $\rho \in \mathbb{R}$, such that

$$
\begin{array}{r}
(1-\lambda) \mathcal{F}\left(u_{1}\right)+\lambda \mathcal{F}\left(u_{2}\right) \subseteq \mathcal{F}\left(\mathcal{H}_{u_{1}, u_{2}}(\lambda)\right)+\rho \lambda(1-\lambda)\left\|u_{1}-u_{2}\right\|^{2} e+\Omega, \\
\forall u_{1}, u_{2} \in A \text { and } \forall \lambda \in[0,1] .
\end{array}
$$

Remark 1. If $\rho>0$, then $\mathcal{F}$ is called strongly $\rho$ - $\Omega$-arcwise connected; if $\rho=0$, we have the notion of $\Omega$-arcwise connectedness; and if $\rho<0$, then $\mathcal{F}$ is called weakly $\rho$ - $\Omega$-arcwise connected. Obviously, strongly $\rho$ - $\Omega$-arcwise connectedness $\Rightarrow \Omega$-arcwise connectedness $\Rightarrow$ weakly $\rho$ - $\Omega$ arcwise connectedness.

Further, we formulate an example of $\rho$-cone arcwise connected set-valued map, which is not cone arcwise connected. 
Example 1. Let $U=\mathbb{R}^{2}, V=\mathbb{R}, \Omega=\mathbb{R}_{+}$and

$$
A=\left\{u=\left(u_{1}, u_{2}\right) \mid u_{1}+u_{2} \geq \frac{1}{2}, u_{1} \geq 0, u_{2} \geq 0\right\} \subset U .
$$

Define $\mathcal{H}_{u, s}(\lambda)=(1-\lambda) u+\lambda s$, where $u=\left(u_{1}, u_{2}\right), s=\left(s_{1}, s_{2}\right)$, and $\lambda \in[0,1]$. Clearly, $A$ is an arcwise connected set. For the set-valued map $\mathcal{F}: U \rightarrow 2^{V}$, defined as follows: $\mathcal{F}(u)=[0,2]$, $u_{1}+u_{2} \geq \frac{1}{2}, u_{1} \neq u_{2}$, and $\mathcal{F}(u)=[3,5]$ for $\left\{u_{1}+u_{2}<\frac{1}{2}\right\} \cup\left\{u_{1}+u_{2} \geq \frac{1}{2}, u_{1}=u_{2}\right\}$, we find that $\mathcal{F}$ is not $\Omega$-arcwise connected for $u=(1,0), s=(0,1)$ and $\lambda=\frac{1}{2}$. On the other hand, by considering $\rho=-2$ and $e=[3,3]=\{3\}$, we get that $\mathcal{F}$ is a $\rho$ - $\Omega$-arcwise connected set-valued map for $u=(1,0), s=(0,1)$.

Theorem 1. Let $A$ be an arcwise connected subset of a real normed space $U, e \in \operatorname{int}(\Omega)$, and $\mathcal{F}: U \rightarrow 2^{V}$ be $\rho$ - $\Omega$-arcwise connected on $A$ with respect to $e$. Let $u^{\prime} \in A$ and $v^{\prime} \in \mathcal{F}\left(u^{\prime}\right)$. Then,

$$
\mathcal{F}(u)-v^{\prime} \subseteq D_{\uparrow} \mathcal{F}\left(u^{\prime}, v^{\prime}\right)\left(\mathcal{H}_{u^{\prime}, u}^{\prime}(0+)\right)+\rho\left\|u-u^{\prime}\right\|^{2} e+\Omega, \quad \forall u \in A,
$$

where

$$
\mathcal{H}_{u^{\prime}, u}^{\prime}(0+)=\lim _{\lambda \rightarrow 0+} \frac{\mathcal{H}_{u^{\prime}, u}(\lambda)-\mathcal{H}_{u^{\prime}, u}(0)}{\lambda},
$$

assuming that $\mathcal{H}_{u^{\prime}, u}^{\prime}(0+)$ exists for all $u, u^{\prime} \in A$.

Proof. Let $u \in A$. As $\mathcal{F}$ is $\rho$ - $\Omega$-arcwise connected on $A$ with respect to $e$, we have

$$
\begin{array}{r}
(1-\lambda) \mathcal{F}\left(u^{\prime}\right)+\lambda \mathcal{F}(u) \subseteq \mathcal{F}\left(\mathcal{H}_{u^{\prime}, u}(\lambda)\right)+\rho \lambda(1-\lambda)\left\|u-u^{\prime}\right\|^{2} e+\Omega, \\
\forall \lambda \in[0,1] .
\end{array}
$$

Let $v \in \mathcal{F}(u)$. Consider a real sequence $\left\{\lambda_{n}\right\}$, with $\lambda_{n} \in(0,1), n \in \mathbb{N}$, such that $\lambda_{n} \rightarrow 0+$ when $n \rightarrow \infty$. Suppose

$$
u_{n}=\mathcal{H}_{u^{\prime}, u}\left(\lambda_{n}\right)
$$

and

$$
v_{n}=\left(1-\lambda_{n}\right) v^{\prime}+\lambda_{n} v-\rho \lambda_{n}\left(1-\lambda_{n}\right)\left\|u-u^{\prime}\right\|^{2} e .
$$

Therefore,

$$
v_{n} \in \mathcal{F}\left(u_{n}\right)+\Omega
$$

It is clear that

$$
\begin{gathered}
u_{n}=\mathcal{H}_{u^{\prime}, u}\left(\lambda_{n}\right) \rightarrow \mathcal{H}_{u^{\prime}, u}(0)=u^{\prime}, v_{n} \rightarrow v^{\prime} \text {, when } n \rightarrow \infty, \\
\frac{u_{n}-u^{\prime}}{\lambda_{n}}=\frac{\mathcal{H}_{u^{\prime}, u}\left(\lambda_{n}\right)-\mathcal{H}_{u^{\prime}, u}(0)}{\lambda_{n}} \rightarrow \mathcal{H}_{u^{\prime}, u}^{\prime}(0+) \text {, when } n \rightarrow \infty,
\end{gathered}
$$

and

$$
\frac{v_{n}-v^{\prime}}{\lambda_{n}}=v-v^{\prime}-\rho\left(1-\lambda_{n}\right)\left\|u-u^{\prime}\right\|^{2} e \rightarrow v-v^{\prime}-\rho\left\|u-u^{\prime}\right\|^{2} e, \text { when } n \rightarrow \infty .
$$

Therefore,

$$
\left(\mathcal{H}_{u^{\prime}, u}^{\prime}(0+), v-v^{\prime}-\rho\left\|u-u^{\prime}\right\|^{2} e\right) \in T\left(\operatorname{epi}(\mathcal{F}),\left(u^{\prime}, v^{\prime}\right)\right)=\operatorname{epi}\left(D_{\uparrow} \mathcal{F}\left(u^{\prime}, v^{\prime}\right)\right) .
$$

Consequently,

$$
v-v^{\prime}-\rho\left\|u-u^{\prime}\right\|^{2} e \in D_{\uparrow} \mathcal{F}\left(u^{\prime}, v^{\prime}\right)\left(\mathcal{H}_{u^{\prime}, u}^{\prime}(0+)\right)+\Omega,
$$


which is true, for all $v \in \mathcal{F}(u)$. Hence,

$$
\mathcal{F}(u)-v^{\prime} \subseteq D_{\uparrow} \mathcal{F}\left(u^{\prime}, v^{\prime}\right)\left(\mathcal{H}_{u^{\prime}, u}^{\prime}(0+)\right)+\rho\left\|u-u^{\prime}\right\|^{2} e+\Omega, \quad \forall u \in A .
$$

Hence, the theorem follows.

\section{Formulation of the Main Problem}

Let $U$ be a countably infinite subset of $\mathbb{R}^{p}, \varnothing \neq A \subseteq \mathbb{R}^{n}$, and $\mathcal{F}=\left(\mathcal{F}_{1}, \mathcal{F}_{2}, \ldots, \mathcal{F}_{m}\right)$ : $\mathbb{R}^{n} \rightarrow 2^{\mathbb{R}^{m}}, \mathcal{G}: \mathbb{R}^{n} \times U \rightarrow 2^{\mathbb{R}}$ be two set-valued maps with

$$
A \subseteq \operatorname{dom}(\mathcal{F}) \text { and } A \times U \subseteq \operatorname{dom}(\mathcal{G}) .
$$

Let $B_{1}, B_{2}, \ldots, B_{m}$ be $n \times n$ positive semi-definite symmetric real matrices. We consider a set-valued semi-infinite programming problem (SP).

$$
\begin{array}{ll}
\underset{x \in A}{\operatorname{minimize}} & \left(\mathcal{F}_{1}(x)+\left(x^{T} B_{1} x\right)^{\frac{1}{2}}, \mathcal{F}_{2}(x)+\left(x^{T} B_{2} x\right)^{\frac{1}{2}}, \ldots, \mathcal{F}_{m}(x)+\left(x^{T} B_{m} x\right)^{\frac{1}{2}}\right) \\
\text { subject to } & \mathcal{G}(x, u) \cap\left(-\mathbb{R}_{+}\right) \neq \varnothing, \forall u \in U .
\end{array}
$$

We define the feasible set of the problem (SP) by

$$
S=\left\{x \in A: \mathcal{G}(x, u) \cap\left(-\mathbb{R}_{+}\right) \neq \varnothing, \forall u \in U\right\} .
$$

Definition 8. A point $\left(x^{\prime}, y^{\prime}\right) \in \mathbb{R}^{n} \times \mathbb{R}^{m}$, with $x^{\prime} \in S$ and $y^{\prime}=\left(y_{1}^{\prime}, y_{2}^{\prime}, \ldots, y_{m}^{\prime}\right) \in \mathcal{F}\left(x^{\prime}\right)$, is said to be a minimizer of the problem (SP) if for all $(x, y) \in \mathbb{R}^{n} \times \mathbb{R}^{m}$, with $x \in S$ and $y=\left(y_{1}, y_{2}, \ldots, y_{m}\right) \in \mathcal{F}(x)$,

$$
\begin{aligned}
& \left(y_{1}+\left(x^{T} B_{1} x\right)^{\frac{1}{2}}, y_{2}+\left(x^{T} B_{2} x\right)^{\frac{1}{2}}, \ldots, y_{m}+\left(x^{T} B_{m} x\right)^{\frac{1}{2}}\right) \\
& -\left(y_{1}^{\prime}+\left(x^{T} B_{1} x^{\prime}\right)^{\frac{1}{2}}, y_{2}^{\prime}+\left(x^{\prime T} B_{2} x^{\prime}\right)^{\frac{1}{2}}, \ldots, y_{m}^{\prime}+\left(x^{\prime T} B_{m} x^{\prime}\right)^{\frac{1}{2}}\right) \notin\left(-\mathbb{R}_{+}^{m}\right) \backslash\left\{\theta_{\mathbb{R}^{m}}\right\} .
\end{aligned}
$$

Definition 9. A point $\left(x^{\prime}, y^{\prime}\right) \in \mathbb{R}^{n} \times \mathbb{R}^{m}$, with $x^{\prime} \in S$ and $y^{\prime}=\left(y_{1}^{\prime}, y_{2}^{\prime} \ldots, y_{m}^{\prime}\right) \in \mathcal{F}\left(x^{\prime}\right)$, is said to be a weak minimizer of the problem (SP) if for all $(x, y) \in \mathbb{R}^{n} \times \mathbb{R}^{m}$, with $x \in S$ and $y=\left(y_{1}, y_{2}, \ldots, y_{m}\right) \in \mathcal{F}(x)$,

$$
\begin{aligned}
& \left(y_{1}+\left(x^{T} B_{1} x\right)^{\frac{1}{2}}, y_{2}+\left(x^{T} B_{2} x\right)^{\frac{1}{2}}, \ldots, y_{m}+\left(x^{T} B_{m} x\right)^{\frac{1}{2}}\right) \\
& -\left(y_{1}^{\prime}+\left(x^{\prime T} B_{1} x^{\prime}\right)^{\frac{1}{2}}, y_{2}^{\prime}+\left(x^{\prime T} B_{2} x^{\prime}\right)^{\frac{1}{2}}, \ldots, y_{m}^{\prime}+\left(x^{\prime T} B_{m} x^{\prime}\right)^{\frac{1}{2}}\right) \notin\left(-\operatorname{int}\left(\mathbb{R}_{+}^{m}\right)\right) .
\end{aligned}
$$

Let $J$ be the index set, such that $U=\left\{u_{j}: j \in J\right\}$. Let $x^{\prime} \in A$. We denote a set $J\left(x^{\prime}\right)$ by

$$
J\left(x^{\prime}\right)=\left\{j \in J: 0 \in \mathcal{G}\left(x^{\prime}, u_{j}\right)\right\} .
$$

We assume that $J\left(x^{\prime}\right) \neq \varnothing$.

For special case, when $f=\left(f_{1}, f_{2}, \ldots, f_{m}\right): \mathbb{R}^{n} \rightarrow \mathbb{R}^{m}$ and $g: \mathbb{R}^{n} \times U \rightarrow \mathbb{R}$ are single-valued maps, we can have multiobjective semi-infinite programming problem ([9]) as

$$
\begin{array}{ll}
\underset{x \in A}{\operatorname{minimize}} & \left(f_{1}(x)+\left(x^{T} B_{1} x\right)^{\frac{1}{2}}, f_{2}(x)+\left(x^{T} B_{2} x\right)^{\frac{1}{2}}, \ldots, f_{m}(x)+\left(x^{T} B_{m} x\right)^{\frac{1}{2}}\right) \\
\text { subject to } & g(x, u) \in\left(-\mathbb{R}_{+}\right), \forall u \in U,
\end{array}
$$

by considering $\mathcal{F}_{i}(x)=\left\{f_{i}(x)\right\}, i=1,2, \ldots, m$, and $\mathcal{G}(x, u)=\{g(x, u)\}$ in the problem (SP).

\section{Optimality Conditions}

Let $\bar{x}_{i} \in \mathbb{R}^{n}, i=1,2, \ldots, m$. Define maps $.{ }^{T} B_{i} \bar{x}_{i}: \mathbb{R}^{n} \rightarrow \mathbb{R}, i=1,2, \ldots, m$, by

$$
\left({ }^{T} B_{i} \bar{x}_{i}\right)(x)=x^{T} B_{i} \bar{x}_{i}, \forall x \in \mathbb{R}^{n} .
$$


The gradient vector of . ${ }^{T} B_{i} \bar{x}_{i}$, denoted by $\nabla\left({ }^{T} B_{i} \bar{x}_{i}\right)$, is given by

$$
\nabla\left({ }^{T} B_{i} \bar{x}_{i}\right)=B_{i} \bar{x}_{i} .
$$

Let $x^{\prime} \in A$ and $j \in J\left(x^{\prime}\right)$. Define a set-valued map $\mathcal{G}\left(., u_{j}\right): \mathbb{R}^{n} \rightarrow 2^{\mathbb{R}}$ by

$$
\mathcal{G}\left(., u_{j}\right)(x)=\mathcal{G}\left(x, u_{j}\right), \forall x \in \operatorname{dom}(\mathcal{G}) .
$$

We establish the sufficient KKT optimality conditions of the set-valued semi-infinite programming problem (SP) under $\rho$-cone arcwise connectedness assumption.

Theorem 2 (Sufficient optimality conditions). Let $A$ be an arcwise connected subset of $\mathbb{R}^{n}$, $x^{\prime} \in S$, and $y^{\prime}=\left(y_{1}^{\prime}, y_{2}^{\prime}, \ldots, y_{m}^{\prime}\right) \in \mathcal{F}\left(x^{\prime}\right)$. Let $\bar{x}_{i} \in \mathbb{R}^{n}, i=1,2, \ldots, m$ and $z^{\prime}=\left(z_{j}^{\prime}\right)_{j \in J}$, with $z_{j}^{\prime} \in \mathcal{G}\left(x^{\prime}, u_{j}\right) \cap\left(-\mathbb{R}_{+}\right)$. Let $\rho_{i}, \rho_{i}^{\prime}, \rho_{j}^{\prime \prime} \in \mathbb{R}$, for $i=1,2, \ldots$, m and $j \in J\left(x^{\prime}\right)$. Suppose that $\mathcal{F}_{i},{ }^{T} B_{i} \bar{x}_{i}, i=1,2, \ldots, m$, and $\mathcal{G}\left(., u_{j}\right), j \in J\left(x^{\prime}\right)$, are $\rho_{i}-\mathbb{R}_{+}$-arcwise connected, $\rho_{i}^{\prime}$ - $\mathbb{R}_{+}$-arcwise connected, and $\rho_{j}^{\prime \prime}-\mathbb{R}_{+}$-arcwise connected set-valued maps, respectively, on $A$ with respect to 1 . Further, we suppose that the contingent epiderivatives $D_{\uparrow} \mathcal{F}_{i}\left(x^{\prime}, y_{i}^{\prime}\right)$ and $D_{\uparrow} \mathcal{G}\left(., u_{j}\right)\left(x^{\prime}, z_{j}^{\prime}\right)$ exist. If there exist $y_{i}^{*}>0, i=1,2, \ldots, m$, and $z_{j}^{*} \geq 0, j \in J\left(x^{\prime}\right)$, with $z_{j}^{*} \neq 0$, for finitely many $j$, and

$$
\sum_{i=1}^{m} y_{i}^{*}\left(\rho_{i}+\rho_{i}^{\prime}\right)+\sum_{j \in J\left(x^{\prime}\right)} z_{j}^{*} \rho_{j}^{\prime \prime} \geq 0,
$$

satisfying the following conditions

$$
\begin{gathered}
\left(\sum_{i=1}^{m} y_{i}^{*}\left(D_{\uparrow} \mathcal{F}_{i}\left(x^{\prime}, y_{i}^{\prime}\right)+\left(B_{i} \bar{x}_{i}\right)^{T}\right)+\sum_{j \in J\left(x^{\prime}\right)} z_{j}^{*} D_{\uparrow} \mathcal{G}\left(., u_{j}\right)\left(x^{\prime}, z_{j}^{\prime}\right)\right)\left(H_{x^{\prime}, x}^{\prime}(0+)\right) \\
\geq 0, \forall x \in A, \\
z_{j}^{*} z_{j}^{\prime}=0, \forall j \in J\left(x^{\prime}\right), \\
\bar{x}_{i}^{T} B_{i} \bar{x}_{i} \leq 1, i=1,2, \ldots, m,
\end{gathered}
$$

and

$$
\left(x^{\prime T} B_{i} x^{\prime}\right)^{\frac{1}{2}}=x^{\prime T} B_{i} \bar{x}_{i}, i=1,2, \ldots, m .
$$

Then, $\left(x^{\prime}, y^{\prime}\right)$ is a weak minimizer of $(S P)$.

Proof. Let $\left(x^{\prime}, y^{\prime}\right)$ be not a weak minimizer of (SP). Then there exist $x \in S$ and $y=$ $\left(y_{1}, \ldots, y_{m}\right) \in \mathcal{F}(x)$, such that

$$
\begin{aligned}
& \left(y_{1}+\left(x^{T} B_{1} x\right)^{\frac{1}{2}}, y_{2}+\left(x^{T} B_{2} x\right)^{\frac{1}{2}}, \ldots, y_{m}+\left(x^{T} B_{m} x\right)^{\frac{1}{2}}\right) \\
< & \left(y_{1}^{\prime}+\left(x^{\prime T} B_{1} x^{\prime}\right)^{\frac{1}{2}}, y_{2}^{\prime}+\left(x^{\prime T} B_{2} x^{\prime}\right)^{\frac{1}{2}}, \ldots, y_{m}^{\prime}+\left(x^{\prime T} B_{m} x^{\prime}\right)^{\frac{1}{2}}\right) .
\end{aligned}
$$

As $y^{*} \in \mathbb{R}_{+}^{m} \backslash\left\{\theta_{\mathbb{R}^{m}}\right\}$, we have

$$
\sum_{i=1}^{m} y_{i}^{*}\left(y_{i}+\left(x^{T} B_{i} x\right)^{\frac{1}{2}}\right)<\sum_{i=1}^{m} y_{i}^{*}\left(y_{i}^{\prime}+\left(x^{\prime T} B_{i} x^{\prime}\right)^{\frac{1}{2}}\right) .
$$

Since $\mathcal{F}_{i}, i=1,2, \ldots, m$, is $\rho_{i}-\mathbb{R}_{+}$-arcwise connected on $A$ with respect to 1 and $\left(x^{\prime}, y_{i}^{\prime}\right) \in \operatorname{gr}\left(\mathcal{F}_{i}\right)$, we have

$$
\mathcal{F}_{i}(x)-y_{i}^{\prime}-\rho_{i}\left\|x-x^{\prime}\right\|^{2} \subseteq D_{\uparrow} \mathcal{F}_{i}\left(x^{\prime}, y_{i}^{\prime}\right)\left(H_{x^{\prime}, x}^{\prime}(0+)\right)+\mathbb{R}_{+} .
$$

Hence,

$$
y_{i}-y_{i}^{\prime}-\rho_{i}\left\|x-x^{\prime}\right\|^{2} \in D_{\uparrow} \mathcal{F}_{i}\left(x^{\prime}, y_{i}^{\prime}\right)\left(H_{x^{\prime}, x}^{\prime}(0+)\right)+\mathbb{R}_{+} .
$$


Therefore,

$$
y_{i}^{*}\left(y_{i}-y_{i}^{\prime}\right)-\rho_{i}\left\|x-x^{\prime}\right\|^{2} y_{i}^{*} \geq y_{i}^{*} D_{\uparrow} \mathcal{F}_{i}\left(x^{\prime}, y_{i}^{\prime}\right)\left(H_{x^{\prime}, x}^{\prime}(0+)\right) .
$$

Again, as $.^{T} B_{i} \bar{x}_{i}, i=1,2, \ldots, m$ and $\mathcal{G}\left(., u_{j}\right), j \in J\left(x^{\prime}\right)$, are $\rho_{i}^{\prime}$ - $\mathbb{R}_{+}$-arcwise connected and $\rho_{j}^{\prime \prime}-\mathbb{R}_{+}$-arcwise connected, respectively, on $A$, with respect to 1 , we have

$$
x^{T} B_{i} \bar{x}_{i}-x^{\prime T} B_{i} \bar{x}_{i}-\rho_{i}^{\prime}\left\|x-x^{\prime}\right\|^{2} \geq\left(B_{i} \bar{x}_{i}\right)^{T}\left(H_{x^{\prime}, x}^{\prime}(0+)\right)
$$

and

$$
\mathcal{G}\left(x, u_{j}\right)-z_{j}^{\prime}-\rho_{j}^{\prime \prime}\left\|x-x^{\prime}\right\|^{2} \subseteq D_{\uparrow} \mathcal{G}\left(., u_{j}\right)\left(x^{\prime}, z_{j}^{\prime}\right)\left(H_{x^{\prime}, x}^{\prime}(0+)\right)+\mathbb{R}_{+} .
$$

As $x \in S$, there exists $z_{j} \in \mathcal{G}\left(x, u_{j}\right) \cap\left(-\mathbb{R}_{+}\right), j \in J\left(x^{\prime}\right)$. So, we have

$$
z_{j}-z_{j}^{\prime}-\rho_{j}^{\prime \prime}\left\|x-x^{\prime}\right\|^{2} \in D_{\uparrow} \mathcal{G}\left(., u_{j}\right)\left(x^{\prime}, z_{j}^{\prime}\right)\left(H_{x^{\prime}, x}^{\prime}(0+)\right)+\mathbb{R}_{+} .
$$

Therefore,

$$
y_{i}^{*}\left(x^{T} B_{i} \bar{x}_{i}-x^{\prime T} B_{i} \bar{x}_{i}\right)-\rho_{i}^{\prime}\left\|x-x^{\prime}\right\|^{2} y_{i}^{*} \geq y_{i}^{*}\left(B_{i} \bar{x}_{i}\right)^{T}\left(H_{x^{\prime}, x}^{\prime}(0+)\right)
$$

and

$$
z_{j}^{*}\left(z_{j}-z_{j}^{\prime}\right)-\rho_{j}^{\prime \prime}\left\|x-x^{\prime}\right\|^{2} z_{j}^{*} \geq z_{j}^{*} D_{\uparrow} \mathcal{G}\left(., u_{j}\right)\left(x^{\prime}, z_{j}^{\prime}\right)\left(H_{x^{\prime}, x}^{\prime}(0+)\right) .
$$

From (7)-(9), we have

$$
\begin{aligned}
& \sum_{i=1}^{m} y_{i}^{*}\left(y_{i}-y_{i}^{\prime}+x^{T} B_{i} \bar{x}_{i}-x^{\prime T} B_{i} \bar{x}_{i}\right)+\sum_{j \in J\left(x^{\prime}\right)} z_{j}^{*}\left(z_{j}-z_{j}^{\prime}\right) \\
& -\left\|x-x^{\prime}\right\|^{2} \sum_{i=1}^{m} y_{i}^{*}\left(\rho_{i}+\rho_{i}^{\prime}\right)-\left\|x-x^{\prime}\right\|^{2} \sum_{j \in J\left(x^{\prime}\right)} z_{j}^{*} \rho_{j}^{\prime \prime} \\
& \geq\left(\sum_{i=1}^{m} y_{i}^{*}\left(D_{\uparrow} \mathcal{F}_{i}\left(x^{\prime}, y_{i}^{\prime}\right)+\left(B_{i} \bar{x}_{i}\right)^{T}\right)+\sum_{j \in J\left(x^{\prime}\right)} z_{j}^{*} D_{\uparrow} \mathcal{G}\left(., u_{j}\right)\left(x^{\prime}, z_{j}^{\prime}\right)\right)\left(H_{x^{\prime}, x}^{\prime}(0+)\right) \geq 0 .
\end{aligned}
$$

From (1), we have

$$
\begin{aligned}
& \sum_{i=1}^{m} y_{i}^{*}\left(y_{i}-y_{i}^{\prime}+x^{T} B_{i} \bar{x}_{i}-x^{\prime T} B_{i} \bar{x}_{i}\right)+\sum_{j \in J\left(x^{\prime}\right)} z_{j}^{*}\left(z_{j}-z_{j}^{\prime}\right) \\
& \geq\left\|x-x^{\prime}\right\|^{2}\left(\sum_{i=1}^{m} y_{i}^{*}\left(\rho_{i}+\rho_{i}^{\prime}\right)+\sum_{j \in J\left(x^{\prime}\right)} z_{j}^{*} \rho_{j}^{\prime \prime}\right) \\
& \geq 0
\end{aligned}
$$

As $z_{j}^{*} z_{j}^{\prime}=0$ and $z_{j} \in\left(-\mathbb{R}_{+}\right), \forall j \in J\left(x^{\prime}\right)$, we have

$$
\sum_{j \in J\left(x^{\prime}\right)} z_{j}^{*}\left(z_{j}-z_{j}^{\prime}\right) \leq 0 .
$$

Hence,

$$
\sum_{i=1}^{m} y_{i}^{*}\left(y_{i}-y_{i}^{\prime}+x^{T} B_{i} \bar{x}_{i}-x^{\prime T} B_{i} \bar{x}_{i}\right) \geq 0 .
$$

Using the generalized Schwarz inequality, we have

$$
\left(x^{T} B_{i} x\right)^{\frac{1}{2}}\left(\bar{x}_{i}^{T} B_{i} \bar{x}_{i}\right)^{\frac{1}{2}} \geq x^{T} B_{i} \bar{x}_{i} .
$$


Again, from (5), we have

$$
\left(x^{\prime T} B_{i} x^{\prime}\right)^{\frac{1}{2}}=x^{\prime T} B_{i} \bar{x}_{i}, i=1,2, \ldots, m .
$$

Therefore,

$$
\sum_{i=1}^{m} y_{i}^{*}\left(y_{i}-y_{i}^{\prime}+\left(x^{T} B_{i} x\right)^{\frac{1}{2}}\left(\bar{x}_{i}^{T} B_{i} \bar{x}_{i}\right)^{\frac{1}{2}}-\left(x^{\prime T} B_{i} x^{\prime}\right)^{\frac{1}{2}}\right) \geq 0 .
$$

From (4), we have $\bar{x}_{i}^{T} B_{i} \bar{x}_{i} \leq 1, i=1,2, \ldots, m$. So, we have

$$
\left(x^{T} B_{i} x\right)^{\frac{1}{2}} \geq\left(x^{T} B_{i} x\right)^{\frac{1}{2}}\left(\bar{x}_{i}^{T} B_{i} \bar{x}_{i}\right)^{\frac{1}{2}} .
$$

Hence,

$$
\sum_{i=1}^{m} y_{i}^{*}\left(y_{i}+\left(x^{T} B_{i} x\right)^{\frac{1}{2}}\right) \geq \sum_{i=1}^{m} y_{i}^{*}\left(y_{i}^{\prime}+\left(x^{\prime T} B_{i} x^{\prime}\right)^{\frac{1}{2}}\right),
$$

which contradicts (6). Hence, $\left(x^{\prime}, y^{\prime}\right)$ is a weak minimizer of (SP).

\section{Mond-Weir Type Dual}

We consider the Mond-Weir type dual (MWD) of the problem (SP), where $\mathcal{F}_{i}$ and $\mathcal{G}\left(., u_{j}\right)$ are contingent epiderivable set-valued maps:

$$
\begin{array}{ll}
\text { maximize } & \left(y_{1}^{\prime}+\left(x^{\prime T} B_{1} \bar{x}_{1}\right), y_{2}^{\prime}+\left(x^{\prime T} B_{2} \bar{x}_{2}\right), \ldots, y_{m}^{\prime}+\left(x^{\prime T} B_{m} \bar{x}_{m}\right)\right) \\
\text { subject to } & \left(\sum_{i=1}^{m} y_{i}^{*}\left(D_{\uparrow} \mathcal{F}_{i}\left(x^{\prime}, y_{i}^{\prime}\right)+\left(B_{i} \bar{x}_{i}\right)^{T}\right)+\sum_{j \in J\left(x^{\prime}\right)} z_{j}^{*} D_{\uparrow} \mathcal{G}\left(., u_{j}\right)\left(x^{\prime}, z_{j}^{\prime}\right)\right)\left(H_{x^{\prime}, x}^{\prime}(0+)\right) \\
& \geq 0, \forall x \in A, \\
& \sum_{j \in J\left(x^{\prime}\right)} z_{j}^{*} z_{j}^{\prime} \geq 0, \\
& \bar{x}_{i}^{T} B_{i} \bar{x}_{i} \leq 1, \bar{x}_{i} \in \mathbb{R}^{n}, i=1,2, \ldots, m, \\
& x^{\prime} \in A, y^{\prime}=\left(y_{1}^{\prime}, y_{2}^{\prime}, \ldots, y_{m}^{\prime}\right) \in \mathcal{F}\left(x^{\prime}\right), z^{\prime}=\left(z_{j}^{\prime}\right)_{j \in J}, z_{j}^{\prime} \in \mathcal{G}\left(x^{\prime}, u_{j}\right), j \in J, \\
& y_{i}^{*}>0, i=1,2, \ldots, m, \sum_{i=1}^{m} y_{i}^{*}=1, z^{*}=\left(z_{j}^{*}\right)_{j \in J}, z_{j}^{*} \geq 0, j \in J, \\
& \text { and } z_{j}^{*} \neq 0, \text { for finitely many } j \in J .
\end{array}
$$

Definition 10. A feasible point $\left(x^{\prime}, y^{\prime}, \bar{x}_{1}, \bar{x}_{2}, \ldots, \bar{x}_{m}, z^{\prime}, y^{*}, z^{*}\right)$ of (MWD) is said to be a weak maximizer of (MWD) if for all feasible points $\left(x, y, \widehat{x}_{1}, \widehat{x}_{2}, \ldots, \widehat{x}_{m}, z, y_{1}^{*}, z_{1}^{*}\right)$ of (MWD),

$$
\begin{aligned}
& \left(y_{1}^{\prime}+\left(x^{\prime T} B_{1} \bar{x}_{1}\right), y_{2}^{\prime}+\left(x^{\prime T} B_{2} \bar{x}_{2}\right), \ldots, y_{m}^{\prime}+\left(x^{\prime T} B_{m} \bar{x}_{m}\right)\right) \\
\nless & \left(y_{1}+\left(x^{T} B_{1} \widehat{x}_{1}\right), y_{2}+\left(x^{T} B_{2} \widehat{x}_{2}\right), \ldots, y_{m}+\left(x^{T} B_{m} \widehat{x}_{m}\right)\right),
\end{aligned}
$$

where $y=\left(y_{1}, y_{2}, \ldots, y_{m}\right), y^{\prime}=\left(y_{1}^{\prime}, y_{2}^{\prime}, \ldots, y_{m}^{\prime}\right) \in \mathbb{R}^{m}$.

Theorem 3 (Weak duality). Let $A$ be an arcwise connected subset of $\mathbb{R}^{n}$ and $x_{0}$ be an element of the feasible set $S$ of $(S P)$. Let $\left(x^{\prime}, y^{\prime}, \bar{x}_{1}, \bar{x}_{2}, \ldots, \bar{x}_{m}, z^{\prime}, y^{*}, z^{*}\right)$ be a feasible solution of (MWD). Let $\rho_{i}, \rho_{i}^{\prime}, \rho_{j}^{\prime \prime} \in \mathbb{R}$, for $i=1,2, \ldots, m$ and $j \in J\left(x^{\prime}\right)$. Suppose that $\mathcal{F}_{i},{ }^{T} B_{i} \bar{x}_{i}, i=1,2, \ldots, m$, and $\mathcal{G}\left(., u_{j}\right), j \in J\left(x^{\prime}\right)$, are $\rho_{i}-\mathbb{R}_{+}$-arcwise connected, $\rho_{i}^{\prime}-\mathbb{R}_{+}$-arcwise connected, and $\rho_{j}^{\prime \prime}-\mathbb{R}_{+}$-arcwise 
connected set-valued maps, respectively, on $A$, with respect to 1, satisfying (5 1). Further, we suppose that the contingent epiderivatives $D_{\uparrow} \mathcal{F}_{i}\left(x^{\prime}, y_{i}^{\prime}\right)$ and $D_{\uparrow} \mathcal{G}\left(., u_{j}\right)\left(x^{\prime}, z_{j}^{\prime}\right)$ exist. Then,

$$
\begin{aligned}
& \left(\mathcal{F}_{1}\left(x_{0}\right)+\left(x_{0}^{T} B_{1} x_{0}\right)^{\frac{1}{2}}, \mathcal{F}_{2}\left(x_{0}\right)+\left(x_{0}^{T} B_{2} x_{0}\right)^{\frac{1}{2}}, \ldots, \mathcal{F}_{m}\left(x_{0}\right)+\left(x_{0}^{T} B_{m} x_{0}\right)^{\frac{1}{2}}\right) \\
& -\left(y_{1}^{\prime}+\left(x^{\prime T} B_{1} \bar{x}_{1}\right), y_{2}^{\prime}+\left(x^{\prime T} B_{2} \bar{x}_{2}\right), \ldots, y_{m}^{\prime}+\left(x^{\prime T} B_{m} \bar{x}_{m}\right)\right) \subseteq \mathbb{R}^{m} \backslash\left(-\operatorname{int}\left(\mathbb{R}_{+}^{m}\right)\right) .
\end{aligned}
$$

Proof. Assume that there is point $y_{i} \in \mathcal{F}_{i}\left(x_{0}\right), i=1,2, \ldots, m$, such that

$$
\begin{aligned}
& \left(y_{1}+\left(x_{0}^{T} B_{1} x_{0}\right)^{\frac{1}{2}}, y_{2}+\left(x_{0}^{T} B_{2} x_{0}\right)^{\frac{1}{2}}, \ldots, y_{m}+\left(x_{0}^{T} B_{m} x_{0}\right)^{\frac{1}{2}}\right) \\
& -\left(y_{1}^{\prime}+\left(x^{\prime T} B_{1} \bar{x}_{1}\right), y_{2}^{\prime}+\left(x^{\prime T} B_{2} \bar{x}_{2}\right), \ldots, y_{m}^{\prime}+\left(x^{T} B_{m} \bar{x}_{m}\right)\right) \in\left(-\operatorname{int}\left(\mathbb{R}_{+}^{m}\right)\right) .
\end{aligned}
$$

As $y^{*} \in \mathbb{R}_{+}^{m} \backslash\left\{\theta_{\mathbb{R}^{m}}\right\}$, we have

$$
\sum_{i=1}^{m} y_{i}^{*}\left(y_{i}+\left(x_{0}^{T} B_{i} x_{0}\right)^{\frac{1}{2}}\right)<\sum_{i=1}^{m} y_{i}^{*}\left(y_{i}^{\prime}+\left(x^{\prime T} B_{i} \bar{x}_{i}\right)\right) .
$$

As $\mathcal{F}_{i}, i=1,2, \ldots, m$, is $\rho_{i}-\mathbb{R}_{+}$-arcwise connected on $A$ with respect to 1 and $\left(x^{\prime}, y_{i}^{\prime}\right) \in \operatorname{gr}\left(\mathcal{F}_{i}\right)$, we have

$$
\mathcal{F}_{i}\left(x_{0}\right)-y_{i}^{\prime}-\rho_{i}\left\|x_{0}-x^{\prime}\right\|^{2} \subseteq D_{\uparrow} \mathcal{F}_{i}\left(x^{\prime}, y_{i}^{\prime}\right)\left(H_{x^{\prime}, x_{0}}^{\prime}(0+)\right)+\mathbb{R}_{+} .
$$

Hence,

$$
y_{i}-y_{i}^{\prime}-\rho_{i}\left\|x_{0}-x^{\prime}\right\|^{2} \in D_{\uparrow} \mathcal{F}_{i}\left(x^{\prime}, y_{i}^{\prime}\right)\left(H_{x^{\prime}, x_{0}}^{\prime}(0+)\right)+\mathbb{R}_{+} .
$$

Therefore,

$$
y_{i}^{*}\left(y_{i}-y_{i}^{\prime}\right)-\rho_{i}\left\|x_{0}-x^{\prime}\right\|^{2} y_{i}^{*} \geq y_{i}^{*} D_{\uparrow} \mathcal{F}_{i}\left(x^{\prime}, y_{i}^{\prime}\right)\left(H_{x^{\prime}, x_{0}}^{\prime}(0+)\right) .
$$

As . ${ }^{T} B_{i} \bar{x}_{i}, i=1,2, \ldots, m$, is $\rho_{i}^{\prime}-\mathbb{R}_{+}$-arcwise connected on $A$ with respect to 1 , we have

$$
x_{0}^{T} B_{i} \bar{x}_{i}-x^{\prime T} B_{i} \bar{x}_{i}-\rho_{i}^{\prime}\left\|x_{0}-x^{\prime}\right\|^{2} \geq\left(B_{i} \bar{x}_{i}\right)^{T}\left(H_{x^{\prime}, x_{0}}^{\prime}(0+)\right) .
$$

Therefore,

$$
y_{i}^{*}\left(x_{0}^{T} B_{i} \bar{x}_{i}-x^{\prime T} B_{i} \bar{x}_{i}\right)-\rho_{i}^{\prime}\left\|x_{0}-x^{\prime}\right\|^{2} y_{i}^{*} \geq y_{i}^{*}\left(B_{i} \bar{x}_{i}\right)^{T}\left(H_{x^{\prime}, x_{0}}^{\prime}(0+)\right) .
$$

Again, as $\mathcal{G}\left(., u_{j}\right), j \in J\left(x^{\prime}\right)$, is $\rho_{j}^{\prime \prime}-\mathbb{R}_{+}$-arcwise connected on $A$ with respect to 1 and $z_{j}^{\prime} \in \mathcal{G}\left(x^{\prime}, u_{j}\right), j \in J\left(x^{\prime}\right)$, we have

$$
\mathcal{G}\left(x_{0}, u_{j}\right)-z_{j}^{\prime}-\rho_{j}^{\prime \prime}\left\|x_{0}-x^{\prime}\right\|^{2} \subseteq D_{\uparrow} \mathcal{G}\left(., u_{j}\right)\left(x^{\prime}, z_{j}^{\prime}\right)\left(H_{x^{\prime}, x_{0}}^{\prime}(0+)\right)+\mathbb{R}_{+} .
$$

As $x_{0} \in S$, there exists $z_{j} \in \mathcal{G}\left(x_{0}, u_{j}\right) \cap\left(-\mathbb{R}_{+}\right), j \in J\left(x^{\prime}\right)$, we have

$$
z_{j}-z_{j}^{\prime}-\rho_{j}^{\prime \prime}\left\|x_{0}-x^{\prime}\right\|^{2} \in D_{\uparrow} \mathcal{G}\left(., u_{j}\right)\left(x^{\prime}, z_{j}^{\prime}\right)\left(H_{x^{\prime}, x_{0}}^{\prime}(0+)\right)+\mathbb{R}_{+} .
$$

Hence,

$$
z_{j}^{*}\left(z_{j}-z_{j}^{\prime}\right)-\rho_{j}^{\prime \prime}\left\|x_{0}-x^{\prime}\right\|^{2} z_{j}^{*} \geq z_{j}^{*} D_{\uparrow} \mathcal{G}\left(., u_{j}\right)\left(x^{\prime}, z_{j}^{\prime}\right)\left(H_{x^{\prime}, x_{0}}^{\prime}(0+)\right) .
$$


From (11)-(13), we have

$$
\begin{aligned}
& \sum_{i=1}^{m} y_{i}^{*}\left(y_{i}-y_{i}^{\prime}+x_{0}^{T} B_{i} \bar{x}_{i}-x^{\prime T} B_{i} \bar{x}_{i}\right)+\sum_{j \in J\left(x^{\prime}\right)} z_{j}^{*}\left(z_{j}-z_{j}^{\prime}\right) \\
& -\left\|x_{0}-x^{\prime}\right\|^{2} \sum_{i=1}^{m} y_{i}^{*}\left(\rho_{i}+\rho_{i}^{\prime}\right)-\left\|x_{0}-x^{\prime}\right\|^{2} \sum_{j \in J\left(x^{\prime}\right)} z_{j}^{*} \rho_{j}^{\prime \prime} \\
& \geq\left(\sum_{i=1}^{m} y_{i}^{*}\left(D_{\uparrow} \mathcal{F}_{i}\left(x^{\prime}, y_{i}^{\prime}\right)+\left(B_{i} \bar{x}_{i}\right)^{T}\right)+\sum_{j \in J\left(x^{\prime}\right)} z_{j}^{*} D_{\uparrow} \mathcal{G}\left(., u_{j}\right)\left(x^{\prime}, z_{j}^{\prime}\right)\right)\left(H_{x^{\prime}, x_{0}}^{\prime}(0+)\right) \\
& \geq 0 .
\end{aligned}
$$

From (1), we have

$$
\begin{aligned}
& \sum_{i=1}^{m} y_{i}^{*}\left(y_{i}-y_{i}^{\prime}+x_{0}^{T} B_{i} \bar{x}_{i}-x^{\prime T} B_{i} \bar{x}_{i}\right)+\sum_{j \in J\left(x^{\prime}\right)} z_{j}^{*}\left(z_{j}-z_{j}^{\prime}\right) \\
& \geq\left\|x_{0}-x^{\prime}\right\|^{2}\left(\sum_{i=1}^{m} y_{i}^{*}\left(\rho_{i}+\rho_{i}^{\prime}\right)+\sum_{j \in J\left(x^{\prime}\right)} z_{j}^{*} \rho_{j}^{\prime \prime}\right) \\
& \geq 0 .
\end{aligned}
$$

As $\sum_{j \in J\left(x^{\prime}\right)} z_{j}^{*} z_{j}^{\prime} \geq 0, z_{j}^{*} \geq 0$, and $z_{j} \in\left(-\mathbb{R}_{+}\right), j \in J\left(x^{\prime}\right)$, we have

$$
\sum_{j \in J\left(x^{\prime}\right)} z_{j}^{*}\left(z_{j}-z_{j}^{\prime}\right) \leq 0
$$

Hence,

$$
\sum_{i=1}^{m} y_{i}^{*}\left(y_{i}-y_{i}^{\prime}+x_{0}^{T} B_{i} \bar{x}_{i}-x^{\prime T} B_{i} \bar{x}_{i}\right) \geq 0 .
$$

Using the generalized Schwarz inequality, we have

$$
\left(x_{0}^{T} B_{i} x_{0}\right)^{\frac{1}{2}}\left(\bar{x}_{i}^{T} B_{i} \bar{x}_{i}\right)^{\frac{1}{2}} \geq x_{0}^{T} B_{i} \bar{x}_{i} .
$$

Again, from the constraints of (MWD), we have

$$
\bar{x}_{i}^{T} B_{i} \bar{x}_{i} \leq 1, i=1,2, \ldots, m .
$$

Hence, $\left(x_{0}^{T} B_{i} x_{0}\right)^{\frac{1}{2}} \geq x_{0}^{T} B_{i} \bar{x}_{i}$. It shows that

$$
\sum_{i=1}^{m} y_{i}^{*}\left(y_{i}+\left(x_{0}^{T} B_{i} x_{0}\right)^{\frac{1}{2}}\right) \geq \sum_{i=1}^{m} y_{i}^{*}\left(y_{i}^{\prime}+\left(x^{\prime T} B_{i} \bar{x}_{i}\right)\right),
$$

which contradicts (10). Therefore,

$$
\begin{aligned}
& \left(y_{1}+\left(x_{0}^{T} B_{1} x_{0}\right)^{\frac{1}{2}}, y_{2}+\left(x_{0}^{T} B_{2} x_{0}\right)^{\frac{1}{2}}, \ldots, y_{m}+\left(x_{0}^{T} B_{m} x_{0}\right)^{\frac{1}{2}}\right) \\
& -\left(y_{1}^{\prime}+\left(x^{\prime T} B_{1} \bar{x}_{1}\right), y_{2}^{\prime}+\left(x^{\prime T} B_{2} \bar{x}_{2}\right), \ldots, y_{m}^{\prime}+\left(x^{\prime T} B_{m} \bar{x}_{m}\right)\right) \notin\left(-\operatorname{int}\left(\mathbb{R}_{+}^{m}\right)\right) .
\end{aligned}
$$

Hence,

$$
\begin{aligned}
& \left(\mathcal{F}_{1}\left(x_{0}\right)+\left(x_{0}^{T} B_{1} x_{0}\right)^{\frac{1}{2}}, \mathcal{F}_{2}\left(x_{0}\right)+\left(x_{0}^{T} B_{2} x_{0}\right)^{\frac{1}{2}}, \ldots, \mathcal{F}_{m}\left(x_{0}\right)+\left(x_{0}^{T} B_{m} x_{0}\right)^{\frac{1}{2}}\right) \\
& -\left(y_{1}^{\prime}+\left(x^{\prime T} B_{1} \bar{x}_{1}\right), y_{2}^{\prime}+\left(x^{\prime T} B_{2} \bar{x}_{2}\right), \ldots, y_{m}^{\prime}+\left(x^{\prime T} B_{m} \bar{x}_{m}\right)\right) \subseteq \mathbb{R}^{m} \backslash\left(-\operatorname{int}\left(\mathbb{R}_{+}^{m}\right)\right) .
\end{aligned}
$$

It completes the proof of the theorem. 
Theorem 4 (Strong duality). Suppose that $\left(x^{\prime}, y^{\prime}\right)$ is a weak minimizer of $(S P), z^{\prime}=\left(z_{j}^{\prime}\right)_{j \in J}$, $z_{j}^{\prime} \in \mathcal{G}\left(x^{\prime}, u_{j}\right) \cap\left(-\mathbb{R}_{+}\right), j \in J$, and $\bar{x}_{i} \in \mathbb{R}^{n}, i=1,2, \ldots, m$. Suppose that, for some $y_{i}^{*}>0$, $i=1,2, \ldots, m$, with $\sum_{i=1}^{m} y_{i}^{*}=1$ and $z_{j}^{*} \geq 0$, with $z_{j}^{*} \neq 0$ for finitely many $j \in J$ and $z_{j}^{*}=0$, $\forall j \in J \backslash J\left(x^{\prime}\right)$. Equations (5.2)-(5.5) are satisfied at the point $\left(x^{\prime}, y^{\prime}, \bar{x}_{1}, \bar{x}_{2}, \ldots, \bar{x}_{m}, z^{\prime}, y^{*}, z^{*}\right)$. Then, $\left(x^{\prime}, y^{\prime}, \bar{x}_{1}, \bar{x}_{2}, \ldots, \bar{x}_{m}, z^{\prime}, y^{*}, z^{*}\right)$ is a feasible solution of (MWD). If the Theorem 6.1 holds between the problems (SP) and (MWD), then $\left(x^{\prime}, y^{\prime}, \bar{x}_{1}, \bar{x}_{2}, \ldots, \bar{x}_{m}, z^{\prime}, y^{*}, z^{*}\right)$ is a weak maximizer of (MWD).

Proof. As (2)-(5) are satisfied at the point $\left(x^{\prime}, y^{\prime}, \bar{x}_{1}, \ldots, \bar{x}_{m}, z^{\prime}, y^{*}, z^{*}\right)$, we have

$$
\begin{gathered}
\left(\sum_{i=1}^{m} y_{i}^{*}\left(D_{\uparrow} \mathcal{F}_{i}\left(x^{\prime}, y_{i}^{\prime}\right)+\left(B_{i} \bar{x}_{i}\right)^{T}\right)+\sum_{j \in J\left(x^{\prime}\right)} z_{j}^{*} D_{\uparrow} \mathcal{G}\left(., u_{j}\right)\left(x^{\prime}, z_{j}^{\prime}\right)\right)\left(\left(H_{x^{\prime}, x}^{\prime}(0+)\right)\right) \\
\geq 0, \forall x \in A, \\
z_{j}^{*} z_{j}^{\prime}=0, \forall j \in J\left(x^{\prime}\right), \\
\bar{x}_{i}^{T} B_{i} \bar{x}_{i} \leq 1, i=1,2, \ldots, m,
\end{gathered}
$$

and

$$
\left(x^{\prime T} B_{i} x^{\prime}\right)^{\frac{1}{2}}=x^{\prime T} B_{i} \bar{x}_{i}, i=1,2, \ldots, m .
$$

Hence, $\left(x^{\prime}, y^{\prime}, \bar{x}_{1}, \bar{x}_{2}, \ldots, \bar{x}_{m}, z^{\prime}, y^{*}, z^{*}\right)$ is a feasible solution of (MWD). Assume that the weak duality Theorem 3 holds between the problems (SP) and (MWD). Let $\left(x^{\prime}, y^{\prime}, \bar{x}_{1}, \bar{x}_{2}, \ldots, \bar{x}_{m}, z^{\prime}, y^{*}, z^{*}\right)$ be not a weak maximizer of (MWD). Then, there exists a feasible solution $\left(x, y, \widehat{x}_{1}, \widehat{x}_{2}, \ldots, \widehat{x}_{m}, z, y_{1}^{*}, z_{1}^{*}\right)$ of (MWD), such that

$$
\begin{aligned}
& \left(y_{1}^{\prime}+\left(x^{\prime T} B_{1} \bar{x}_{1}\right), y_{2}^{\prime}+\left(x^{\prime T} B_{2} \bar{x}_{2}\right), \ldots, y_{m}^{\prime}+\left(x^{\prime T} B_{m} \bar{x}_{m}\right)\right) \\
& -\left(y_{1}+\left(x^{T} B_{1} \widehat{x}_{1}\right), y_{2}+\left(x^{T} B_{2} \widehat{x}_{2}\right), \ldots, y_{m}+\left(x^{T} B_{m} \widehat{x}_{m}\right)\right) \in\left(-\operatorname{int}\left(\mathbb{R}_{+}^{m}\right)\right) .
\end{aligned}
$$

where $y=\left(y_{1}, \ldots, y_{m}\right) \in \mathbb{R}^{m}$. As $\left(x^{\prime T} B_{i} x^{\prime}\right)^{\frac{1}{2}}=x^{\prime T} B_{i} \bar{x}_{i}, i=1,2, \ldots, m$, we have

$$
\begin{aligned}
& \left(y_{1}^{\prime}+\left(x^{\prime T} B_{1} x^{\prime}\right)^{\frac{1}{2}}, y_{2}^{\prime}+\left(x^{\prime T} B_{2} x^{\prime}\right)^{\frac{1}{2}}, \ldots, y_{m}^{\prime}+\left(x^{\prime T} B_{m} x^{\prime}\right)^{\frac{1}{2}}\right) \\
& -\left(y_{1}+\left(x^{T} B_{1} \widehat{x}_{1}\right), y_{2}+\left(x^{T} B_{2} \widehat{x}_{2}\right), \ldots, y_{m}+\left(x^{T} B_{m} \widehat{x}_{m}\right)\right) \in\left(-\operatorname{int}\left(\mathbb{R}_{+}^{m}\right)\right),
\end{aligned}
$$

which contradicts the Theorem 3 between (SP) and (MWD). Hence, $\left(x^{\prime}, y^{\prime}, \bar{x}_{1}, \ldots, \bar{x}_{m}, z^{\prime}\right.$, $\left.y^{*}, z^{*}\right)$ is a weak maximizer of (MWD).

Theorem 5 (Converse duality). Let $A$ be an arcwise connected subset of $\mathbb{R}^{n}$. Suppose that $\left(x^{\prime}, y^{\prime}, \bar{x}_{1}, \bar{x}_{2}, \ldots, \bar{x}_{m}, z^{\prime}, y^{*}, z^{*}\right)$ is a feasible solution of (MWD), with

$$
\left(x^{\prime T} B_{i} x^{\prime}\right)^{\frac{1}{2}}=x^{\prime T} B_{i} \bar{x}_{i}, i=1,2, \ldots, m .
$$

Let $\rho_{i}, \rho_{i}^{\prime}, \rho_{j}^{\prime \prime} \in \mathbb{R}$, for $i=1,2, \ldots, m$ and $j \in J\left(x^{\prime}\right)$. Suppose that $\mathcal{F}_{i}, .{ }^{T} B_{i} \bar{x}_{i}, i=1,2, \ldots, m$, and $\mathcal{G}\left(., u_{j}\right), j \in J\left(x^{\prime}\right)$, are $\rho_{i}-\mathbb{R}_{+}$-arcwise connected, $\rho_{i}^{\prime}-\mathbb{R}_{+}$-arcwise connected, and $\rho_{j}^{\prime \prime}-\mathbb{R}_{+}$-arcwise connected set-valued maps, respectively, on $A$, with respect to 1 , satisfying (1). We suppose that the contingent epiderivatives $D_{\uparrow} \mathcal{F}_{i}\left(x^{\prime}, y_{i}^{\prime}\right)$ and $D_{\uparrow} \mathcal{G}\left(., u_{j}\right)\left(x^{\prime}, z_{j}^{\prime}\right)$ exist. If $x^{\prime} \in S$, then $\left(x^{\prime}, y^{\prime}\right)$ is a weak minimizer of (SP).

Proof. Let $\left(x^{\prime}, y^{\prime}\right)$ be not a weak minimizer of (SP). Then, $x \in S$ and $y=\left(y_{1}, y_{2}, \ldots, y_{m}\right) \in \mathcal{F}(x)$ exist, such that

$$
\begin{aligned}
& \left(y_{1}+\left(x^{T} B_{1} x\right)^{\frac{1}{2}}, y_{2}+\left(x^{T} B_{2} x\right)^{\frac{1}{2}}, \ldots, y_{m}+\left(x^{T} B_{m} x\right)^{\frac{1}{2}}\right) \\
& -\left(y_{1}^{\prime}+\left(x^{\prime T} B_{1} x^{\prime}\right)^{\frac{1}{2}}, y_{2}^{\prime}+\left(x^{\prime T} B_{2} x^{\prime}\right)^{\frac{1}{2}}, \ldots, y_{m}^{\prime}+\left(x^{\prime T} B_{m} x^{\prime}\right)^{\frac{1}{2}}\right) \in\left(-\operatorname{int}\left(\mathbb{R}_{+}^{m}\right)\right) .
\end{aligned}
$$


As $y^{*} \in \mathbb{R}_{+}^{m} \backslash\left\{\theta_{\mathbb{R}^{m}}\right\}$, we have

$$
\sum_{i=1}^{m} y_{i}^{*}\left(y_{i}+\left(x^{T} B_{i} x\right)^{\frac{1}{2}}\right)<\sum_{i=1}^{m} y_{i}^{*}\left(y_{i}^{\prime}+\left(x^{\prime T} B_{i} x^{\prime}\right)^{\frac{1}{2}}\right) .
$$

Since $\mathcal{F}_{i}, i=1,2, \ldots, m$, is $\rho_{i}-\mathbb{R}_{+}$-arcwise connected on $A$ with respect to 1 and $\left(x^{\prime}, y_{i}^{\prime}\right) \in \operatorname{gr}\left(\mathcal{F}_{i}\right)$, we have

$$
\mathcal{F}_{i}(x)-y_{i}^{\prime}-\rho_{i}\left\|x-x^{\prime}\right\|^{2} \subseteq D_{\uparrow} \mathcal{F}_{i}\left(x^{\prime}, y_{i}^{\prime}\right)\left(H_{x^{\prime}, x}^{\prime}(0+)\right)+\mathbb{R}_{+} .
$$

Hence,

$$
y_{i}-y_{i}^{\prime}-\rho_{i}\left\|x-x^{\prime}\right\|^{2} \in D_{\uparrow} \mathcal{F}_{i}\left(x^{\prime}, y_{i}^{\prime}\right)\left(H_{x^{\prime}, x}^{\prime}(0+)\right)+\mathbb{R}_{+} .
$$

Therefore,

$$
y_{i}^{*}\left(y_{i}-y_{i}^{\prime}\right)-\rho_{i}\left\|x-x^{\prime}\right\|^{2} y_{i}^{*} \geq y_{i}^{*} D_{\uparrow} \mathcal{F}_{i}\left(x^{\prime}, y_{i}^{\prime}\right)\left(H_{x^{\prime}, x}^{\prime}(0+)\right) .
$$

Again, as $.{ }^{T} B_{i} \bar{x}_{i}, i=1,2, \ldots, m$, and $\mathcal{G}\left(., u_{j}\right), j \in J\left(x^{\prime}\right)$, are $\rho_{i}^{\prime}-\mathbb{R}_{+}$-arcwise connected and $\rho_{j}^{\prime \prime}-\mathbb{R}_{+}$-arcwise connected, respectively, on $A$, with respect to 1 , we have

$$
x^{T} B_{i} \bar{x}_{i}-x^{\prime T} B_{i} \bar{x}_{i}-\rho_{i}^{\prime}\left\|x-x^{\prime}\right\|^{2} \geq\left(B_{i} \bar{x}_{i}\right)^{T}\left(H_{x^{\prime}, x}^{\prime}(0+)\right)
$$

and

$$
\mathcal{G}\left(x, u_{j}\right)-z_{j}^{\prime}-\rho_{j}^{\prime \prime}\left\|x-x^{\prime}\right\|^{2} \subseteq D_{\uparrow} \mathcal{G}\left(., u_{j}\right)\left(x^{\prime}, z_{j}^{\prime}\right)\left(H_{x^{\prime}, x}^{\prime}(0+)\right)+\mathbb{R}_{+} .
$$

As $x \in S$, there exists $z_{j} \in \mathcal{G}\left(x, u_{j}\right) \cap\left(-\mathbb{R}_{+}\right), \forall j \in J\left(x^{\prime}\right)$. So,

$$
z_{j}-z_{j}^{\prime}-\rho_{j}^{\prime \prime}\left\|x-x^{\prime}\right\|^{2} \in D_{\uparrow} \mathcal{G}\left(., u_{j}\right)\left(x^{\prime}, z_{j}^{\prime}\right)\left(H_{x^{\prime}, x}^{\prime}(0+)\right)+\mathbb{R}_{+} .
$$

Therefore,

$$
y_{i}^{*}\left(x^{T} B_{i} \bar{x}_{i}-x^{\prime T} B_{i} \bar{x}_{i}\right)-\rho_{i}^{\prime}\left\|x-x^{\prime}\right\|^{2} y_{i}^{*} \geq y_{i}^{*}\left(B_{i} \bar{x}_{i}\right)^{T}\left(H_{x^{\prime}, x}^{\prime}(0+)\right)
$$

and

$$
z_{j}^{*}\left(z_{j}-z_{j}^{\prime}\right)-\rho_{j}^{\prime \prime}\left\|x-x^{\prime}\right\|^{2} z_{j}^{*} \geq z_{j}^{*} D_{\uparrow} \mathcal{G}\left(., u_{j}\right)\left(x^{\prime}, z_{j}^{\prime}\right)\left(H_{x^{\prime}, x}^{\prime}(0+)\right) .
$$

From (15)-(17), we have

$$
\begin{aligned}
& \sum_{i=1}^{m} y_{i}^{*}\left(y_{i}-y_{i}^{\prime}+x^{T} B_{i} \bar{x}_{i}-x^{\prime T} B_{i} \bar{x}_{i}\right)+\sum_{j \in J\left(x^{\prime}\right)} z_{j}^{*}\left(z_{j}-z_{j}^{\prime}\right) \\
& -\left\|x-x^{\prime}\right\|^{2} \sum_{i=1}^{m} y_{i}^{*}\left(\rho_{i}+\rho_{i}^{\prime}\right)-\left\|x-x^{\prime}\right\|^{2} \sum_{j \in J\left(x^{\prime}\right)} z_{j}^{*} \rho_{j}^{\prime \prime} \\
& \geq\left(\sum_{i=1}^{m} y_{i}^{*}\left(D_{\uparrow} \mathcal{F}_{i}\left(x^{\prime}, y_{i}^{\prime}\right)+\left(B_{i} \bar{x}_{i}\right)^{T}\right)+\sum_{j \in J\left(x^{\prime}\right)} z_{j}^{*} D_{\uparrow} \mathcal{G}\left(., u_{j}\right)\left(x^{\prime}, z_{j}^{\prime}\right)\right)\left(H_{x^{\prime}, x}^{\prime}(0+)\right) \geq 0 .
\end{aligned}
$$

By (1), we have

$$
\begin{aligned}
& \sum_{i=1}^{m} y_{i}^{*}\left(y_{i}-y_{i}^{\prime}+x^{T} B_{i} \bar{x}_{i}-x^{\prime T} B_{i} \bar{x}_{i}\right)+\sum_{j \in J\left(x^{\prime}\right)} z_{j}^{*}\left(z_{j}-z_{j}^{\prime}\right) \\
& \geq\left\|x-x^{\prime}\right\|^{2}\left(\sum_{i=1}^{m} y_{i}^{*}\left(\rho_{i}+\rho_{i}^{\prime}\right)+\sum_{j \in J\left(x^{\prime}\right)} z_{j}^{*} \rho_{j}^{\prime \prime}\right) \\
& \geq 0 .
\end{aligned}
$$


As $\sum_{j \in J\left(x^{\prime}\right)} z_{j}^{*} z_{j}^{\prime} \geq 0, z_{j}^{*} \geq 0$, and $z_{j} \in\left(-\mathbb{R}_{+}\right), j \in J\left(x^{\prime}\right)$, we have

$$
\sum_{j \in J\left(x^{\prime}\right)} z_{j}^{*}\left(z_{j}-z_{j}^{\prime}\right) \leq 0
$$

Hence,

$$
\sum_{i=1}^{m} y_{i}^{*}\left(y_{i}-y_{i}^{\prime}+x^{T} B_{i} \bar{x}_{i}-x^{\prime T} B_{i} \bar{x}_{i}\right) \geq 0 .
$$

From the generalized Schwarz inequality, we have

$$
\left(x^{T} B_{i} x\right)^{\frac{1}{2}}\left(\bar{x}_{i}^{T} B_{i} \bar{x}_{i}\right)^{\frac{1}{2}} \geq x^{T} B_{i} \bar{x}_{i} .
$$

Again, by assumption, we have

$$
\left(x^{\prime T} B_{i} x^{\prime}\right)^{\frac{1}{2}}=x^{\prime T} B_{i} \bar{x}_{i}, i=1,2, \ldots, m .
$$

Therefore,

$$
\sum_{i=1}^{m} y_{i}^{*}\left(y_{i}-y_{i}^{\prime}+\left(x^{T} B_{i} x\right)^{\frac{1}{2}}\left(\bar{x}_{i}^{T} B_{i} \bar{x}_{i}\right)^{\frac{1}{2}}-\left(x^{\prime T} B_{i} x^{\prime}\right)^{\frac{1}{2}}\right) \geq 0 .
$$

As $\bar{x}_{i}^{T} B_{i} \bar{x}_{i} \leq 1, i=1,2, \ldots, m$, (from the constraints of (MWD)), we have

$$
\left(x^{T} B_{i} x\right)^{\frac{1}{2}} \geq\left(x^{T} B_{i} x\right)^{\frac{1}{2}}\left(\bar{x}_{i}^{T} B_{i} \bar{x}_{i}\right)^{\frac{1}{2}} .
$$

Hence,

$$
\sum_{i=1}^{m} y_{i}^{*}\left(y_{i}+\left(x^{T} B_{i} x\right)^{\frac{1}{2}}\right) \geq \sum_{i=1}^{m} y_{i}^{*}\left(y_{i}^{\prime}+\left(x^{\prime T} B_{i} x^{\prime}\right)^{\frac{1}{2}}\right),
$$

which contradicts (14). So, $\left(x^{\prime}, y^{\prime}\right)$ is a weak minimizer of (SP).

\section{Wolfe Type Dual}

We consider the Wolfe type dual (WD) of the problem (SP), where $\mathcal{F}_{i}$ and $\mathcal{G}\left(., u_{j}\right)$ are contingent epiderivable set-valued maps:

maximize $\quad\left(y_{1}^{\prime}+\left(x^{\prime T} B_{1} \bar{x}_{1}\right), y_{2}^{\prime}+\left(x^{\prime T} B_{2} \bar{x}_{2}\right), \ldots, y_{m}^{\prime}+\left(x^{\prime T} B_{m} \bar{x}_{m}\right)\right)+\left(\sum_{j \in J\left(x^{\prime}\right)} z_{j}^{*} z_{j}^{\prime}\right) \mathbf{1}_{\mathbb{R}^{m}}$

subject to

$$
\begin{aligned}
& \left(\sum_{i=1}^{m} y_{i}^{*}\left(D_{\uparrow} \mathcal{F}_{i}\left(x^{\prime}, y_{i}^{\prime}\right)+\left(B_{i} \bar{x}_{i}\right)^{T}\right)+\sum_{j \in J\left(x^{\prime}\right)} z_{j}^{*} D_{\uparrow} \mathcal{G}\left(., u_{j}\right)\left(x^{\prime}, z_{j}^{\prime}\right)\right)\left(H_{x^{\prime}, x}^{\prime}(0+)\right) \\
& \geq 0, \forall x \in A, \\
& \bar{x}_{i}^{T} B_{i} \bar{x}_{i} \leq 1, \bar{x}_{i} \in \mathbb{R}^{n}, i=1,2, \ldots, m, \\
& x^{\prime} \in A, y^{\prime}=\left(y_{1}^{\prime}, y_{2}^{\prime}, \ldots, y_{m}^{\prime}\right) \in \mathcal{F}\left(x^{\prime}\right), z^{\prime}=\left(z_{j}^{\prime}\right)_{j \in J\left(x^{\prime}\right)}, z_{j}^{\prime} \in \mathcal{G}\left(x^{\prime}, u_{j}\right), j \in J, \\
& y_{i}^{*}>0, i=1,2, \ldots, m, \sum_{i=1}^{m} y_{i}^{*}=1, \text { and } z^{*}=\left(z_{j}^{*}\right)_{j \in J}, z_{j}^{*} \geq 0, j \in J,
\end{aligned}
$$$$
\text { and } z_{j}^{*} \neq 0 \text {, for finitely many } j \in J \text {. }
$$ 
Definition 11. A feasible point $\left(x^{\prime}, y^{\prime}, \bar{x}_{1}, \bar{x}_{2}, \ldots, \bar{x}_{m}, z^{\prime}, y^{*}, z^{*}\right)$ of $(W D)$ is said to be a weak maximizer of $(W D)$ if for all feasible points $\left(x, y, \widehat{x}_{1}, \widehat{x}_{2}, \ldots, \widehat{x}_{m}, z, y_{1}^{*}, z_{1}^{*}\right)$ of (WD),

$$
\begin{aligned}
& \left(y_{1}^{\prime}+\left(x^{\prime T} B_{1} \bar{x}_{1}\right), y_{2}^{\prime}+\left(x^{\prime T} B_{2} \bar{x}_{2}\right), \ldots, y_{m}^{\prime}+\left(x^{\prime T} B_{m} \bar{x}_{m}\right)\right)+\left(\sum_{j \in J\left(x^{\prime}\right)} z_{j}^{*} z_{j}^{\prime}\right) \mathbf{1}_{\mathbb{R}^{m}} \\
& \nless\left(y_{1}+\left(x^{T} B_{1} \widehat{x}_{1}\right), y_{2}+\left(x^{T} B_{2} \widehat{x}_{2}\right), \ldots, y_{m}+\left(x^{T} B_{m} \widehat{x}_{m}\right)\right)+\left(\sum_{j \in J\left(x^{\prime}\right)} z_{j}^{*} z_{j}\right) \mathbf{1}_{\mathbb{R}^{m}},
\end{aligned}
$$

where $y=\left(y_{1}, y_{2}, \ldots, y_{m}\right), y^{\prime}=\left(y_{1}^{\prime}, y_{2}^{\prime}, \ldots, y_{m}^{\prime}\right) \in \mathbb{R}^{m}$.

We can prove the duality theorems of Wolfe type associated with the problem (SP). The proofs are very similar to Theorems $3-5$, and hence we omit it.

Theorem 6 (Weak duality). Let $A$ be an arcwise connected subset of $\mathbb{R}^{n}$ and $x_{0} \in S$. Let $\left(x^{\prime}, y^{\prime}, \bar{x}_{1}, \bar{x}_{2}, \ldots, \bar{x}_{m}, z^{\prime}, y^{*}, z^{*}\right)$ be a feasible solution of $(W D)$. Let $\rho_{i}, \rho_{i}^{\prime}, \rho_{j}^{\prime \prime} \in \mathbb{R}$, for $i=1,2, \ldots, m$ and $j \in J\left(x^{\prime}\right)$. Suppose that $\mathcal{F}_{i}, \cdot{ }^{T} B_{i} \bar{x}_{i}, i=1,2, \ldots, m$, and $\mathcal{G}\left(., u_{j}\right), j \in J\left(x^{\prime}\right)$, are $\rho_{i}-\mathbb{R}_{+}$-arcwise connected, $\rho_{i}^{\prime}-\mathbb{R}_{+}$-arcwise connected, and $\rho_{j}^{\prime \prime}-\mathbb{R}_{+}$-arcwise connected set-valued maps, respectively, on $A$, with respect to 1 , satisfying (1). Suppose that the contingent epiderivatives $D_{\uparrow} \mathcal{F}_{i}\left(x^{\prime}, y_{i}^{\prime}\right)$ and $D_{\uparrow} \mathcal{G}\left(., u_{j}\right)\left(x^{\prime}, z_{j}^{\prime}\right)$ exist. Then,

$$
\begin{array}{r}
\left(\mathcal{F}_{1}\left(x_{0}\right)+\left(x_{0}^{T} B_{1} x_{0}\right)^{\frac{1}{2}}, \mathcal{F}_{2}\left(x_{0}\right)+\left(x_{0}^{T} B_{2} x_{0}\right)^{\frac{1}{2}}, \ldots, \mathcal{F}_{m}\left(x_{0}\right)+\left(x_{0}^{T} B_{m} x_{0}\right)^{\frac{1}{2}}\right) \\
\nless\left(y_{1}^{\prime}+\left(x^{\prime T} B_{1} \bar{x}_{1}\right), y_{2}^{\prime}+\left(x^{\prime T} B_{2} \bar{x}_{2}\right), \ldots, y_{m}^{\prime}+\left(x^{\prime T} B_{m} \bar{x}_{m}\right)\right)+\left(\sum_{j \in J\left(x^{\prime}\right)} z_{j}^{*} z_{j}^{\prime}\right) \mathbf{1}_{\mathbb{R}^{m}} .
\end{array}
$$

Theorem 7 (Strong duality). Suppose that $\left(x^{\prime}, y^{\prime}\right)$ is a weak minimizer of $(S P), z^{\prime}=\left(z_{j}^{\prime}\right)_{j \in J}$, $z_{j}^{\prime} \in \mathcal{G}\left(x^{\prime}, u_{j}\right) \cap\left(-\mathbb{R}_{+}\right), j \in J$, and $\bar{x}_{i} \in \mathbb{R}^{n}, i=1,2, \ldots, m$. Suppose that for some $y_{i}^{*}>0$, $i=1,2, \ldots, m$, with $\sum_{i=1}^{m} y_{i}^{*}=1$ and $z_{j}^{*} \geq 0$, with $z_{j}^{*} \neq 0$ for finitely many $j \in J$ and $z_{j}^{*}=0$, $\forall j \in J \backslash J\left(x^{\prime}\right)$, Equations (2)-(5) are satisfied at the point $\left(x^{\prime}, y^{\prime}, \bar{x}_{1}, \bar{x}_{2}, \ldots, \bar{x}_{m}, z^{\prime}, y^{*}, z^{*}\right)$. Then, $\left(x^{\prime}, y^{\prime}, \bar{x}_{1}, \ldots, \bar{x}_{m}, z^{\prime}, y^{*}, z^{*}\right)$ is a feasible solution of (WD). If the Theorem 6 holds between the problems (SP) and (WD), then $\left(x^{\prime}, y^{\prime}, \bar{x}_{1}, \bar{x}_{2}, \ldots, \bar{x}_{m}, z^{\prime}, y^{*}, z^{*}\right)$ is a weak maximizer of (WD).

Theorem 8 (Converse duality). Let $A$ be an arcwise connected subset of $\mathbb{R}^{n}$. Suppose that $\left(x^{\prime}, y^{\prime}, \bar{x}_{1}, \bar{x}_{2}, \ldots, \bar{x}_{m}, z^{\prime}, y^{*}, z^{*}\right)$ is a feasible solution of (WD), with

$$
\left(x^{\prime T} B_{i} x^{\prime}\right)^{\frac{1}{2}}=x^{\prime T} B_{i} \bar{x}_{i}, i=1,2, \ldots, m
$$

and

$$
\sum_{j \in J\left(x^{\prime}\right)} z_{j}^{*} z_{j}^{\prime} \geq 0
$$

Let $\rho_{i}, \rho_{i}^{\prime}, \rho_{j}^{\prime \prime} \in \mathbb{R}$, for $i=1,2, \ldots$, m and $j \in J\left(x^{\prime}\right)$. Suppose that $\mathcal{F}_{i},{ }^{T} B_{i} \bar{x}_{i}, i=1,2, \ldots, m$, and $\mathcal{G}\left(., u_{j}\right), j \in J\left(x^{\prime}\right)$, are $\rho_{i}-\mathbb{R}_{+}$-arcwise connected, $\rho_{i}^{\prime}-\mathbb{R}_{+}$-arcwise connected, and $\rho_{j}^{\prime \prime}-\mathbb{R}_{+}$-arcwise connected set-valued maps, respectively, on $A$, with respect to 1 , satisfying (1). We suppose that the contingent epiderivatives $D_{\uparrow} \mathcal{F}_{i}\left(x^{\prime}, y_{i}^{\prime}\right)$ and $D_{\uparrow} \mathcal{G}\left(., u_{j}\right)\left(x^{\prime}, z_{j}^{\prime}\right)$ exist. If $x^{\prime} \in S$, then $\left(x^{\prime}, y^{\prime}\right)$ is a weak minimizer of (SP). 


\section{Mixed Type Dual}

We consider the mixed type dual (MD) of the problem (SP), where $\mathcal{F}_{i}$ and $\mathcal{G}\left(., u_{j}\right)$ are contingent epiderivable set-valued maps:

maximize

$$
\left(y_{1}^{\prime}+\left(x^{\prime T} B_{1} \bar{x}_{1}\right), y_{2}^{\prime}+\left(x^{\prime T} B_{2} \bar{x}_{2}\right), \ldots, y_{m}^{\prime}+\left(x^{\prime T} B_{m} \bar{x}_{m}\right)\right)+\left(\sum_{j \in J\left(x^{\prime}\right)} z_{j}^{*} z_{j}^{\prime}\right) \mathbf{1}_{\mathbb{R}^{m}}
$$

subject to

$$
\begin{aligned}
& \left(\sum_{i=1}^{m} y_{i}^{*}\left(D_{\uparrow} \mathcal{F}_{i}\left(x^{\prime}, y_{i}^{\prime}\right)+\left(B_{i} \bar{x}_{i}\right)^{T}\right)+\sum_{j \in J\left(x^{\prime}\right)} z_{j}^{*} D_{\uparrow} \mathcal{G}\left(., u_{j}\right)\left(x^{\prime}, z_{j}^{\prime}\right)\right)\left(H_{x^{\prime}, x}^{\prime}(0+)\right) \\
& \geq 0, \forall x \in A, \\
& z_{j}^{*} z_{j}^{\prime} \geq 0, \forall j \in J\left(x^{\prime}\right), \\
& \bar{x}_{i}^{T} B_{i} \bar{x}_{i} \leq 1, \bar{x}_{i} \in \mathbb{R}^{n}, i=1,2, \ldots, m, \\
& x^{\prime} \in A, y^{\prime}=\left(y_{1}^{\prime}, y_{2}^{\prime}, \ldots, y_{m}^{\prime}\right) \in \mathcal{F}\left(x^{\prime}\right), z^{\prime}=\left(z_{j}^{\prime}\right)_{j \in J}, z_{j}^{\prime} \in \mathcal{G}\left(x^{\prime}, u_{j}\right), j \in J, \\
& y_{i}^{*}>0, i=1,2, \ldots, m, \sum_{i=1}^{m} y_{i}^{*}=1, \text { and } z^{*}=\left(z_{j}^{*}\right)_{j \in J}, z_{j}^{*} \geq 0, j \in J, \\
& \text { and } z_{j}^{*} \neq 0, \text { for finitely many } j \in J .
\end{aligned}
$$

Definition 12. A feasible point $\left(x^{\prime}, y^{\prime}, \bar{x}_{1}, \bar{x}_{2}, \ldots, \bar{x}_{m}, z^{\prime}, y^{*}, z^{*}\right)$ of $(M D)$ is said to be a weak maximizer of $(M D)$ if for all feasible points $\left(x, y, \widehat{x}_{1}, \widehat{x}_{2}, \ldots, \widehat{x}_{m}, z, y_{1}^{*}, z_{1}^{*}\right)$ of $(M D)$,

$$
\begin{aligned}
& \left(y_{1}^{\prime}+\left(x^{\prime T} B_{1} \bar{x}_{1}\right), y_{2}^{\prime}+\left(x^{\prime T} B_{2} \bar{x}_{2}\right), \ldots, y_{m}^{\prime}+\left(x^{\prime T} B_{m} \bar{x}_{m}\right)\right)+\left(\sum_{j \in J\left(x^{\prime}\right)} z_{j}^{*} z_{j}^{\prime}\right) \mathbf{1}_{\mathbb{R}^{m}} \\
& \nless\left(y_{1}+\left(x^{T} B_{1} \widehat{x}_{1}\right), y_{2}+\left(x^{T} B_{2} \widehat{x}_{2}\right), \ldots, y_{m}+\left(x^{T} B_{m} \widehat{x}_{m}\right)\right)+\left(\sum_{j \in J\left(x^{\prime}\right)} z_{j}^{*} z_{j}\right) \mathbf{1}_{\mathbb{R}^{m}},
\end{aligned}
$$

where $y=\left(y_{1}, y_{2}, \ldots, y_{m}\right), y^{\prime}=\left(y_{1}^{\prime}, y_{2}^{\prime}, \ldots, y_{m}^{\prime}\right) \in \mathbb{R}^{m}$

We develop the duality results of mixed type of the problem (SP). The proofs are very similar to Theorems 3-5, and hence we omit it.

Theorem 9 (Weak duality). Let $A$ be an arcwise connected subset of $\mathbb{R}^{n}$ and $x_{0} \in S$. Let $\left(x^{\prime}, y^{\prime}, \bar{x}_{1}, \bar{x}_{2}, \ldots, \bar{x}_{m}, z^{\prime}, y^{*}, z^{*}\right)$ be a feasible solution of $(M D)$. Let $\rho_{i}, \rho_{i}^{\prime}, \rho_{j}^{\prime \prime} \in \mathbb{R}$, for $i=1,2, \ldots, m$ and $j \in J\left(x^{\prime}\right)$. Suppose that $\mathcal{F}_{i},{ }^{T} B_{i} \bar{x}_{i}, i=1,2, \ldots, m$, and $\mathcal{G}\left(., u_{j}\right), j \in J\left(x^{\prime}\right)$, are $\rho_{i}-\mathbb{R}_{+}{ }^{-}$ arcwise connected, $\rho_{i}^{\prime}-\mathbb{R}_{+}$-arcwise connected, and $\rho_{j}^{\prime \prime}-\mathbb{R}_{+}$-arcwise connected set-valued maps, respectively, on $A$, with respect to 1 , satisfying (1). Let the contingent epiderivatives $D_{\uparrow} \mathcal{F}_{i}\left(x^{\prime}, y_{i}^{\prime}\right)$ and $D_{\uparrow} \mathcal{G}\left(., u_{j}\right)\left(x^{\prime}, z_{j}^{\prime}\right)$ exist. Then,

$$
\begin{aligned}
& \left(\mathcal{F}_{1}\left(x_{0}\right)+\left(x_{0}^{T} B_{1} x_{0}\right)^{\frac{1}{2}}, \mathcal{F}_{2}\left(x_{0}\right)+\left(x_{0}^{T} B_{2} x_{0}\right)^{\frac{1}{2}}, \ldots, \mathcal{F}_{m}\left(x_{0}\right)+\left(x_{0}^{T} B_{m} x_{0}\right)^{\frac{1}{2}}\right) \\
& \nless\left(y_{1}^{\prime}+\left(x^{\prime T} B_{1} \bar{x}_{1}\right), y_{2}^{\prime}+\left(x^{\prime T} B_{2} \bar{x}_{2}\right), \ldots, y_{m}^{\prime}+\left(x^{\prime T} B_{m} \bar{x}_{m}\right)\right)+\left(\sum_{j \in J\left(x^{\prime}\right)} z_{j}^{*} z_{j}^{\prime}\right) \mathbf{1}_{\mathbb{R}^{m}} .
\end{aligned}
$$

Theorem 10 (Strong duality). Suppose that $\left(x^{\prime}, y^{\prime}\right)$ is a weak minimizer of $(S P), z^{\prime}=\left(z_{j}^{\prime}\right)_{j \in J}$, $z_{j}^{\prime} \in \mathcal{G}\left(x^{\prime}, u_{j}\right) \cap\left(-\mathbb{R}_{+}\right), j \in J$, and $\bar{x}_{i} \in \mathbb{R}^{n}, i=1,2, \ldots, m$. Suppose that for some $y_{i}^{*}>0$, $i=1,2, \ldots, m$, with $\sum_{i=1}^{m} y_{i}^{*}=1$ and $z_{j}^{*} \geq 0$, with $z_{j}^{*} \neq 0$ for finitely many $j \in J$ and $z_{j}^{*}=0$, $\forall j \in J \backslash J\left(x^{\prime}\right)$. Equations (2)-(5) are satisfied at the point $\left(x^{\prime}, y^{\prime}, \bar{x}_{1}, \bar{x}_{2}, \ldots, \bar{x}_{m}, z^{\prime}, y^{*}, z^{*}\right)$. Then, $\left(x^{\prime}, y^{\prime}, \bar{x}_{1}, \bar{x}_{2}, \ldots, \bar{x}_{m}, z^{\prime}, y^{*}, z^{*}\right)$ is a feasible solution of $(M D)$. If the Theorem 9 holds between the problems $(S P)$ and $(M D)$, then

$$
\left(x^{\prime}, y^{\prime}, \bar{x}_{1}, \bar{x}_{2}, \ldots, \bar{x}_{m}, z^{\prime}, y^{*}, z^{*}\right)
$$


is a weak maximizer of $(M D)$.

Theorem 11 (Converse duality). Let $A$ be an arcwise connected subset of $\mathbb{R}^{n}$. Suppose that $\left(x^{\prime}, y^{\prime}, \bar{x}_{1}, \bar{x}_{2}, \ldots, \bar{x}_{m}, z^{\prime}, y^{*}, z^{*}\right)$ is a feasible solution of $(M D)$, with $\left(x^{\prime T} B_{i} x^{\prime}\right)^{\frac{1}{2}}=x^{\prime T} B_{i} \bar{x}_{i}$, $i=1,2, \ldots, m$. Let $\rho_{i}, \rho_{i}^{\prime}, \rho_{j}^{\prime \prime} \in \mathbb{R}$, for $i=1,2, \ldots, m$ and $j \in J\left(x^{\prime}\right)$. Suppose that $\mathcal{F}_{i},{ }^{T} B_{i} \bar{x}_{i}$, $i=1,2, \ldots, m$, and $\mathcal{G}\left(., u_{j}\right), j \in J\left(x^{\prime}\right)$, are $\rho_{i}-\mathbb{R}_{+}$-arcwise connected, $\rho_{i}^{\prime}-\mathbb{R}_{+}$-arcwise connected, and $\rho_{j}^{\prime \prime}-\mathbb{R}_{+}$-arcwise connected set-valued maps, respectively, on $A$, with respect to 1 , satisfying (1). We suppose that the contingent epiderivatives $D_{\uparrow} \mathcal{F}_{i}\left(x^{\prime}, y_{i}^{\prime}\right)$ and $D_{\uparrow} \mathcal{G}\left(., u_{j}\right)\left(x^{\prime}, z_{j}^{\prime}\right)$ exist. If $x^{\prime} \in S$, then $\left(x^{\prime}, y^{\prime}\right)$ is a weak minimizer of $(S P)$.

\section{Conclusions}

In this paper, we have established the sufficient KKT optimality conditions of a setvalued semi-infinite programming problem (SP) under $\rho$-cone arcwise connectedness assumptions. We studied the duality theorems of Mond-Weir, Wolfe, and mixed types associated with the problem (SP).

Author Contributions: Conceptualization, K.D.; methodology, K.D. and S.T.; validation, K.D. and S.T. All authors have read and agreed to the published version of the manuscript.

Funding: This research received no external funding.

Institutional Review Board Statement: Not applicable.

Informed Consent Statement: Not applicable.

Data Availability Statement: Not applicable.

Acknowledgments: The authors would like to thank anonymous referees for their careful reading.

Conflicts of Interest: The authors declare no conflict of interest.

\section{References}

1. Hanson, M.A. On sufficiency of the Kuhn-Tucker conditions. J. Math. Anal. Appl. 1981, 80, 545-550. [CrossRef]

2. Craven, B.D. Invex functions and constrained local minima. Bull. Austral. Math. Soc. 1981, 24, 357-366. [CrossRef]

3. Corley, H.W. Existence and Lagrangian duality for maximizations of set-valued functions. J. Optim. Theory Appl. 1987, 54, 489-501. [CrossRef]

4. Zalmai, G.J. Generalized sufficiency criteria in continuous-time programming with application to a class of variational-type inequalities. J. Math. Anal. Appl. 1990, 153, 331-355. [CrossRef]

5. Goberna, M.A.; Lopez, M.A. Linear semi-infinite programming theory: An updated survey. Eur. J. Oper. Res. 2002, 143, 390-405. [CrossRef]

6. Shapiro, A. Semi-infinite programming, duality, discretization and optimality condition. Optimization 2009, 58, 133-161. [CrossRef]

7. Shapiro, A. On duality theory of convex semi-infinite programming. Optimization 2005, 54, 535-543. [CrossRef]

8. Kostyukova, O.I.; Tchemisova, T.V. Sufficient optimality conditions for convex semi-infinite programming. Optim. Methods Softw. 2010, 25, 279-297. [CrossRef]

9. Mishra, S.K.; Jaiswal, M. Optimality conditions and duality for nondifferentiable multiobjective semi-infinite programming. Vietnam J. Math. 2012, 40, 331-343.

10. Avriel, M. Nonlinear Programming: Theory and Method; Prentice-Hall: Englewood Cliffs, NJ, USA, 1976.

11. Fu, J.; Wang, Y. Arcwise connected cone-convex functions and mathematical programming. J. Optim. Theory Appl. 2003, 118, 339-352. [CrossRef]

12. Lalitha, C.; Dutta, J.; Govil, M.G. Optimality criteria in set-valued optimization. J. Aust. Math. Soc. 2003, 75, 221-232. [CrossRef]

13. Yu, G. Optimality of global proper efficiency for cone-arcwise connected set-valued optimization using contingent epiderivative. Asia-Pac. J. Oper. Res. 2013, 30, 1340004. [CrossRef]

14. Yihong, X.; Min, L. Optimality conditions for weakly efficient elements of set-valued optimization with $\alpha$-order near cone-arcwise connectedness. J. Syst. Sci. Math. Sci. 2016, 36, 1721.

15. Yu, G. Global proper efficiency and vector optimization with cone-arcwise connected set-valued maps. Numer. Algebra Control Optim. 2016, 6, 35. [CrossRef]

16. Peng, Z.; Xu, Y. Second-order optimality conditions for cone-subarcwise connected set-valued optimization problems. Acta Math Appl. Sin. Engl. Ser. 2018, 34, 183-196. [CrossRef]

17. Aubin, J.P. Contingent derivatives of set-valued maps and existence of solutions to nonlinear inclusions and differential inclusions. In Mathematical Analysis and Applications, Part A; Nachbin, L., Ed.; Academic Press: New York, NY, USA, 1981; pp. 160-229. 
18. Aubin, J.P.; Frankowska, H. Set-Valued Analysis; Birhäuser: Boston, MA, USA, 1990.

19. Cambini, A.; Martein, L.; Vlach, M. Second order tangent sets and optimality conditions. Math. Jpn. 1999, 49, 451-461.

20. Jahn, J.; Rauh, R. Contingent epiderivatives and set-valued optimization. Math. Method Oper. Res. 1997, 46, 193-211. [CrossRef]

21. Borwein, J. Multivalued convexity and optimization: A unified approach to inequality and equality constraints. Math. Program. 1977, 13, 183-199. [CrossRef]

22. Das, K.; Nahak, C. Approximate quasi efficiency of set-valued optimization problems via weak subdifferential. SeMA J. 2017, 74, 523-542. [CrossRef]

23. Das, K.; Nahak, C. Set-valued minimax programming problems under generalized cone convexity. Rend. Circ. Mat. Palermo 2017, 66, 361-374. [CrossRef]

24. Das, K.; Nahak, C. Sufficiency and duality of set-valued semi-infinite programming problems under generalized cone convexity. Acta Univ. Matthiae Belii Ser. Math. 2020, 2020, 95-111.

25. Das, K.; Nahak, C. Set-valued optimization problems via second-order contingent epiderivative. Yugosl. J. Oper. Res. 2021, 31, 75-94. [CrossRef]

26. Treanta, S.; Das, K. On robust saddle-point criterion in optimization problems with curvilinear integral functionals. Mathematics 2021, 9, 1790. [CrossRef] 